\title{
Le plaisir des femmes selon Aristote
}

Averroès contre Galien sur Natura nihil facit frustra

\section{Cristina Cerami}

\section{(2) OpenEdition}

Journals

Édition électronique

URL : https://journals.openedition.org/philosant/597

DOI : 10.4000/philosant.597

ISSN : 2648-2789

Éditeur

Éditions Vrin

\section{Édition imprimée}

Date de publication : 1 novembre 2016

Pagination : 63-102

ISBN : 978-2-7574-1472-9

ISSN : $1634-4561$

Référence électronique

Cristina Cerami, "Le plaisir des femmes selon Aristote », Philosophie antique [En ligne], 16 | 2016, mis en ligne le 01 novembre 2018, consulté le 02 décembre 2022. URL : http://journals.openedition.org/ philosant/597 ; DOI : https://doi.org/10.4000/philosant.597

\section{(c)}

Creative Commons - Attribution - Pas d'Utilisation Commerciale - Pas de Modification 4.0 International - CC BY-NC-ND 4.0

https://creativecommons.org/licenses/by-nc-nd/4.0/ 


\section{LE PLAISIR DES FEMMES SELON ARISTOTE Averroès contre Galien sur Natura nibil facit frustra Cristina CERAMI \\ CNRS, UMR 7219-SPHERE}

RÉSUMÉ. Cet article se propose d'étudier le phénomène biologique du plaisir sexuel féminin, dans le but d'en déterminer la place dans le cadastre étiologique d'Aristote. En s'appuyant sur une série de textes tirés du De Generatione Animalium, l'auteur suggère que le plaisir sexuel féminin fait partie des phénomènes qu'Aristote définit comme « en vue du meilleur ». L'étude de ce phénomène donne ainsi l'occasion de s'interroger à nouveaux frais sur la place de la téléologie dans le système causal d'Aristote et la nature de la finalité dite « dérivée ». Dans une seconde partie de l'étude, l'auteur propose un aperçu de la réception gréco-arabe de la question. L'étude du débat engagé par Averroès contre Galien au XII ${ }^{\mathrm{e}}$ siècle de notre ère montre l'importance de la question du plaisir sexuel féminin dans le péripatétisme gréco-arabe et permet d'éclairer en retour la doctrine du Stagirite.

SUMMARY. This article is devoted to the biological phenomenon of female sexual pleasure and aims at determining its causal role in Aristotle's biological doctrine. In considering several passages of the De Generatione Animalium, the author suggests that female sexual pleasure is one of the phenomena that Aristotle defines as "for what is better". The study of this phenomenon provides the opportunity to rethink the place of the final cause in Aristotle's causalsystem and the nature of the so-called "derivative" teleology. In the second part of the study, the author provides an overview of the Greco-Arabic reception of Aristotle's doctrine. The study of the debate prompted by Averroes against Galen in the XII $I^{\text {th }}$ century AD shows the importance of the issue of female sexual pleasure in the Greco-Arabic peripatetism and clarifies in turn the doctrine of the Stagyrite.

Philosophie antique, $\mathrm{n}^{\circ} 16$ (2016), 63-102 



\section{Introduction*}

Depuis la seconde moitié du siècle passé, la question de l'existence d'un plaisir sexuel féminin, de sa nature et de sa fonction, s'est progressivement installée au cœur des débats les plus enflammés de la biologie contemporaine. Avant même de devenir de grande actualité dans les gender studies, elle a été à l'origine, dans la biologie évolutionniste, d'une littérature tentaculaire qui a vu les adaptionistes s'opposer aux pluralistes. Le problème, dans le cadre d'une théorie de l'évolution, était d'expliquer l'existence d'une jouissance féminine étant donné que la reproduction a lieu, dans la plupart des cas, sans qu'aucun plaisir ne se produise chez la femme ${ }^{1}$.

La question de la nature et de la raison d'être du plaisir féminin ne naît, toutefois, ni avec la biologie évolutionniste, ni plus généralement avec la biologie moderne, car elle était déjà très débattue dans le monde grec et au Moyen-Âge arabe et latin, notamment dans le milieu médical ${ }^{2}$. De fait, aucun penseur, ni dans le milieu médical ni parmi les philosophes, n'a jamais au sens strict nié l'existence de ce phénomène ; leurs divergences portaient en revanche sur sa nature et sa fonction. En effet, le cœur du débat a toujours été de savoir si le plaisir sexuel féminin possède ou non une nature équivalente à celle du plaisir masculin et un rôle analogue à celui que ce dernier occupe dans la procréation.

* Cette étude a été présentée pour la première fois en 2013 lors du séminaire interéquipes Le plaisir dans les traditions platonicienne et aristotélicienne, organisé à Paris par A. Jaulin, M. Crubellier et moi-même, puis au séminaire Causes et Principes de l'Antiquité au Moyen-Age, organisé la même année par C. Viano et A. Vasiliu. Je remercie les participants aux deux séminaires, et notamment D. Lefebvre, pour leurs remarques et suggestions, ainsi que J. Chandelier, J.-B. Gourinat, T. Bénatouil, et l'auteur/e de la relecture anonyme, qui m'ont tous permis de rendre plus clair le sens de mon propos.

1. Pour une présentation de ce débat et des différentes positions, je renvoie à la monographie d'Elizabeth Lloyd (Lloyd 2005) et à la bibliographie citée.

2. Sur le Moyen-Âge latin, voir Jacquart \& Thomasset 1985. 
C'est dans ce cadre aussi que, dans le De generatione animalium, Aristote $s$ 'interroge sur la nature du plaisir sexuel en général et sur celui des femmes en particulier. Contre les tenants de la théorie de la pangenèse d'une part et de la double semence d'autre part, Aristote formule une doctrine qui s'oppose à toute théorie d'une équivalence stricte entre le plaisir sexuel des deux sexes. Sans jamais y consacrer une étude particulière, Aristote admet l'existence d'un plaisir féminin, comme d'un plaisir masculin, mais précise que seul le second phénomène est véritablement nécessaire à la procréation.

Nombreuses sont les études récentes consacrées à la place que la femelle en général, et la femme en particulier, occupent dans la théorie aristotélicienne de la génération animale. Beaucoup d'entre elles s'interrogent sur le type d'explication causale qu'Aristote en fournit et défendent l'idée que l'existence de la femme et la contribution de celle-ci dans la procréation ne découlent pas de la pure nécessité matérielle, mais qu'elles peuvent être expliquées à la lumière de la doctrine téléologique d'Aristote 3 . Le grand mérite de ces études a été en ce sens de montrer que dans la théorie aristotélicienne de la génération, il ne s'agit pas simplement de départager la cause formalo-finale de la cause matérielle pour ranger l'existence et la fonction de la femme sous cette dernière, mais de montrer que la différentiation des sexes répond chez Aristote à l'exigence d'établir des niveaux de finalité différents dans ses explications biologiques.

Dans cette littérature, toutefois, la place réservée au phénomène biologique du plaisir sexuel féminin demeure très limitée, et sa valeur causale à évaluer ${ }^{4}$. Je me propose dans cette étude d'examiner ce phénomène, dans le but précis d'en déterminer la place dans le cadastre étiologique d'Aristote ${ }^{5}$. Dans ce cadre, et en m'appuyant sur une série de textes tirés du $G A$, je montrerai qu'Aristote propose pour le plaisir féminin comme pour le plaisir masculin une véritable explication causale, mais qu'il envisage pour chacun des deux un type de causalité différente. En effet, même s'il n'accorde pas au plaisir

3. Voir notamment, en plus des travaux cités dans le corps de l'article, Mayhew 2004 ; Lefebvre 2014a ; Bianchi 2014 ; Kosman 2010.

4. En effet, la plupart des études qui discutent du plaisir sexuel féminin ne s'interrogent pas sur la place que celui-ci occupe dans la doctrine aristotélicienne des causes, mais l'envisagent d'un point de vue, pour ainsi dire, physiologique (voir à ce propos Campese, Manuli \& Sissa 1983, p. 120-130, en particulier p. 125 où Giulia Sissa se contente d'affirmer que pour Aristote le liquide qui se produit parfois lors des rapports sexuels, auquel elle donne la fonction d'un lubrifiant, est « un evento accessorio, di un'accidentalità utile, frequente, ma non tassativo »). Ayant envoyé cet article en 2015, je n'ai pas pu tenir compte de l'étude récente de Sophia M. Connell (Aristotle on Female Animals : A Study of the Generation of Animals, Cambridge, 2016, Cambridge Classical Studies). Je remercie le lecteur anonyme de mavoir signalé sa parution récente.

5. Je ne me propose pas en ce sens de répondre à la question générale de savoir ce qu'est le plaisir en tant que tel et quelles en sont les causes. Sur cette question, je renvoie à létude fouillée et novatrice de Campbell 2011. 
sexuel de la femme une forme de nécessité absolue, il en fournit une véritable raison d'être qui n'est pas de l'ordre de la pure nécessité matérielle. Son existence, en effet, n’est ni purement nécessaire ni purement mécanique, mais « en vue du mieux ». Je voudrais en effet suggérer que le plaisir sexuel féminin fait partie des phénomènes qu'on peut expliquer, d'après Aristote, comme le produit d'une finalité relative ou dérivée : il existe non pas en raison d'une nécessité absolue, mais parce qu'il vaut mieux que ce soit ainsi. En cela même, le phénomène du plaisir sexuel féminin confirme l'existence d'une « hiérarchisation » dans l'explication téléologique d'Aristote. L'enjeu de la théorie aristotélicienne des causes n'est en ce sens pas simplement d'articuler la causalité finale à la causalité matérielle, en départageant les phénomènes qui relèveraient de l'une de ceux qui relèveraient de l'autre, mais de dégager à l'intérieur même de la finalité une place pour tous les phénomènes biologiques qui, sans être nécessaires à la survie de l'animal, contribuent médiatement à son complet accomplissement ${ }^{6}$.

Dans une seconde partie de ce travail, je donnerai un aperçu de la réception gréco-arabe du débat concernant la même question. Dans ce cadre aussi, on verra que la question concernant l'existence d'un plaisir sexuel féminin engage une réflexion plus générale sur la cohérence du système téléologique d'Aristote et sur la nature des explications des phénomènes biologiques considérés comme non nécessaires. Le débat qu'au XII siècle de l'ère chrétienne Averroès engage contre Galien constitue un pan essentiel de l'histoire de cette réflexion. On verra que l'existence du plaisir sexuel féminin fournit à Averroès l'occasion de critiquer et réfuter la théorie galénique de la double semence, tout en tirant parti des nouvelles découvertes anatomiques de son prédécesseur.

\section{Plaisir sexuel et causalité chez Aristote}

Pour Aristote, comme pour les savants, médecins et philosophes, qui avant et après lui se sont interrogés sur le plaisir féminin, la question n'a jamais été de prouver l'existence de ce phénomène ${ }^{7}$. Il s'agissait, pour les

6. De nombreuses études sont consacrées à l'existence d'une finalité « relative » dans la biologie aristotélicienne. J'y reviendrai dans le corps de l'article. Pour l'instant, je me contente de signaler que la lecture que je vais en proposer va à l'encontre des interprétations qui font des phénomènes en vue du mieux une forme de « téléologie de second ordre » qui n'opérerait pas sur le potentiel véhiculé par la forme (pour utiliser la célèbre expression de A. Gotthelf « a preexisting potential for form »), mais sur le potentiel que la matière constitutive se trouve posséder.

7. Mon but étant de comprendre la fonction que le plaisir féminin occupe dans la biologie aristotélicienne, je ne chercherai pas à dresser un tableau exhaustif de tous les philosophes et médecins qui se sont penchés sur la question avant et après Aristote. Je me contenterai de considérer les théories des interlocuteurs de l'aristotélisme sur cette question : en amont, la tradition hippocratique et, en aval, les théories galéniques. Pour la même raison, ces théories 
uns comme pour les autres, d'en comprendre la nature et la raison d'être. Cette réflexion partait, en ce sens, du constat de l'existence du plaisir sexuel féminin, comme de celle du liquide qui parfois l'accompagne, considéré comme un résidu qui d'une façon ou d'une autre est lié à la production du plaisir. Leurs divergences, comme on le verra, portaient en revanche sur la nature et la supposée fonction du plaisir féminin et le rôle de ce résidu. De fait, tout le débat concernant la question de l'existence d'un plaisir sexuel féminin reposait en dernière instance sur la nature que les différentes théories attribuaient à ce résidu et à son lien avec la procréation ${ }^{8}$. En effet, la question était de savoir si le fait d'attribuer aux plaisirs de l'homme et de la femme une nature équivalente impliquait nécessairement le fait de leur attribuer la même fonction : la production d'un liquide spermatique nécessaire à la procréation, à savoir une semence 9 . Pour le dire autrement, la question était de déterminer si le plaisir chez la femme, et la production concomitante d'un liquide spermatique, étaient le signe du fait qu'elle contribue à la procréation à la façon dont l'homme le fait, c'est-à-dire en donnant une semence. C'est notamment contre la validité de l'inférence qui remonte de l'existence du plaisir sexuel à la production d'une semence qu'Aristote s'attaque à la fois aux défenseurs de la théorie de la pangenèse et à ceux de la double semence : le fait qu'un plaisir sexuel se produise n'est pas le signe du fait qu'il y a une contribution séminale. Tout l'effort d'Aristote sera alors de montrer, d'abord, que tous les liquides produits au moment du plaisir ne sont pas fertiles, puis d'expliquer la raison pour laquelle une telle émission se produit en concomitance avec le plaisir. Ce n'est une fois expliqué le lien entre la production de ce résidu et le plaisir qu'on pourra donner la raison ultime de ce dernier, chez l'homme comme chez la femme.

\section{1. Plaisir, résidu, procréation : contre la théorie de la pangenèse}

L'idée que le plaisir sexuel soit pour les tenants de la théorie pangénétique un « signe » de la production d'un liquide séminal semble suggérée par la façon dont Aristote critique l'un des arguments en faveur de cette théorie : celui du plaisir, pour ainsi dire, «total ». C'est de cette façon qu'en $G A$ I 18 Aristote présente cet argument, qui consiste à conclure que la semence

ne constituent pas en tant que telles l'objet premier d'analyse du présent article ; elles y sont examinées dans la mesure où elles ont constitué le repoussoir, pour Aristote comme pour Averroès, de leur propre théorie du plaisir féminin.

8. Mon analyse du processus de la génération est en ce sens subordonnée à celle de la question du plaisir sexuel. Pour plus de détails sur la doctrine aristotélicienne de la génération animale, je me permets de renvoyer à l'étude que j'y ai consacrée dans Cerami 2015, p. 129-164, ainsi qu'à la bibliographie citée.

9. J'utilise l'expression « liquide spermatique » pour désigner toute humidité résiduelle produite en concomitance avec l'acte sexuel. J'utiliserai en revanche le mot « semence » ou « liquide séminal » pour désigner tout liquide fertile nécessaire à la procréation. 
procède de toutes les parties du corps, parce que le plaisir qui accompagne sa formation puis son expulsion concerne le corps dans son ensemble :

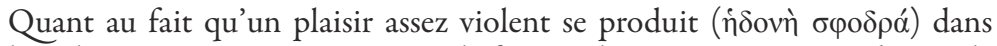
les relations érotiques, ce n'est pas le fait que le sperme provienne de tout le corps qui en est la cause, mais c'est que le chatouillement est violent (

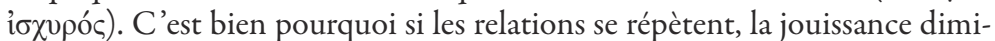
nue pour ceux qui s'y adonnent. En plus, la jouissance ( $\chi \alpha \rho \alpha$ ) arrive à la fin

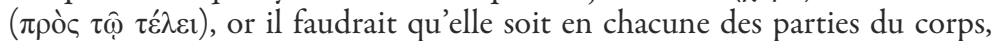
et non d'un seul coup, mais d'abord dans les unes, ensuite dans les autres ${ }^{10}$.

Avant même de considérer la critique qu'Aristote adresse à cet argument, il faut souligner que ce dernier se fonde sur l'assertion d'un lien nécessaire entre le plaisir et la production d'une semence et sur l'idée que le plaisir total est preuve du fait que la semence est produite par l'ensemble du corps. Aristote n'attribue cet argument à aucun penseur en particulier ${ }^{11}$. Mais on sait que la théorie pangénétique a été défendue et développée dans la tradition hippocratique. On en trouve trace dans le traité De la génération attribué à Hippocrate ${ }^{12}$, dans lequel l'existence d'un lien direct entre le plaisir et la production d'une semence est explicitement affirmée et dans lequel cette même inférence est tirée dans le cas du plaisir de la femme ${ }^{13}$. Selon ce traité, que l'on situe aux alentours de la fin du $\mathrm{V}^{\mathrm{e}}$ siècle avant J.-C., toutes les parties du corps produisent de la semence, même si celle-ci est expulsée, à la fin de l'acte sexuel, par une seule d'entre elles. En effet, la semence dont l'expulsion produit la jouissance se concentre dans cette partie, mais elle est produite, en quantité plus ou moins importante, par l'ensemble des parties du corps.

Au début du traité l'auteur explique que lorsque l'organe sexuel est frotté et réchauffé par l'accouplement, il se produit dans ses conduits « comme une démangeaison » (๕̋ $\sigma \varepsilon \rho \kappa v \eta \sigma \mu o ́ c)$, ayant comme effet la diffusion dans tout le corps de plaisir ( $\dot{\eta} \delta o v \eta ́)$ et de chaleur :

La loi gouverne tout; quant au sperme de l'homme il vient de toute l'humeur qui se trouve dans le corps; c'en est la partie la plus forte qui s'est séparée. La preuve que c'est la partie la plus forte qui se sépare, c'est que, après le coït, nous devenons faibles, pour avoir éjaculé une si petite quantité. Voici ce qui en est. Des veines et des nerfs vont du corps entier au sexe; quand ce dernier est frotté, échauffé, rempli, il survient comme une démangeaison, ce qui fournit à tout le corps plaisir et chaleur ${ }^{14}$. (Trad. R. Joly.)

10. Aristote, $G A \mathrm{I} 18,723 \mathrm{~b} 32-724$ a3. Les traductions du $G A$, sauf indications contraires, sont celles de Lefebvre 2014b.

11. Pour une présentation plus détaillée des doctrines critiquées par Aristote, voir Louguet 2015.

12. Morsink 1982, p. 68 sq. fait également le rapprochement entre le traité d'Aristote et le traité pseudo-hippocratique.

13. Sur la théorie hippocratique du plaisir sexuel féminin, voir Dean-Jones 1992.

14. Hippocrate, De la Génération (Genit.), I 1(Joly 1970 = Littré VII, p. 470, 1-10). 
L'auteur explique ainsi que le frottement de l'organe génital produit une chaleur et une démangeaison très vive qui se répandent dans tout le corps. Cet échauffement est à l'origine de la production du sperme à partir de l'ensemble de l'humeur chauffée. En effet, la semence n'est qu'une partie plus puissante et plus grasse qui se sépare de l'humeur qui se trouve dans tout le corps. Elle se sépare, dit la suite du texte, comme l'écume qui se forme sur les corps agités. Elle circule à l'intérieur du corps grâce à un réseau de veines et de nerfs qui recouvre tout le corps et, en partant du cerveau et de la moelle épinière, elle sort à travers l'organe génital dans lequel les nerfs et les veines se terminent.

Le plaisir sexuel accompagne donc la formation d'un liquide séminal et la jouissance finale, l'expulsion d'un concentré de ce même liquide. Mais l'organe sexuel n'est pas la seule partie engagée dans le phénomène du plaisir ; celui-ci, en effet, intéresse tout le corps. Par conséquent, tout le corps produit une semence. Ce processus, précise ensuite l'auteur du traité, concerne l'homme autant que la femme $\mathrm{e}^{15}$ : cette dernière produit et éjacule elle aussi le même type de liquide, considéré comme une semence provenant de tout le corps :

Chez les femmes, le sexe étant frotté dans le coït et la matrice en mouvement, je dis que cette dernière est saisie comme d'une démangeaison (ڤ̋ $\sigma \pi \varepsilon \rho$ $\kappa v \eta \sigma \mu o ̀ v)$ qui apporte plaisir et chaleur au reste du corps. La femme aussi éjacule à partir de (tout) le corps, tantôt dans la matrice - et la matrice devient humide - tantôt en dehors, si la matrice est plus béante qu'il ne convient. Et la femme connaît la jouissance dès le début, pendant tout le temps du coït, jusqu'à ce que l'homme la lâche. Si elle éprouve l'orgasme, elle éjacule avant l'homme et sa jouissance n'est plus la même ; si elle n'éprouve pas l'orgasme sa jouissance finit avec celle de l'homme ${ }^{16}$.

Selon la théorie hippocratique, le plaisir sexuel, qu'il soit féminin ou masculin, est donc dû à une démangeaison globale, ainsi qu'au frottement et il s'achève lors de l'excrétion d'une semence. C'est en effet au moment de l'expulsion que, dans l'homme comme dans la femme, se produit la jouissance. Ce qui sépare les deux sexes, explique alors l'auteur, n'est pas tellement la nature de leur plaisir, mais sa durée et sa violence. La femme, dit-il, connaît la jouissance dès le début et pendant tout le temps, mais son plaisir est moins fort que celui de l'homme. La raison en est que l'excrétion provenant de l'humeur se fait chez lui brusquement, par un trouble plus violent que chez la femme :

Il en va comme lorsqu'on verse du vin sur une flamme : il arrive tout d'abord que la flamme s'élance et augmente quand on verse du vin, puis elle finit. De même chez la femme, la chaleur monte au contact du sperme de l'homme,

15. Genit. IV 1 (Joly 1970).

16. Genit. IV 1, p. 46, 21-47, 6 (Joly 1970). 
puis elle finit. La jouissance de la femme dans le coït est beaucoup moins forte que celle de l'homme, mais elle dure plus longtemps que celle de l'homme. Pourquoi l'homme éprouve-t-il plus de jouissance ? C'est que l'excrétion provenant de l'humeur se fait chez lui brusquement, par un trouble plus violent que chez la femme ${ }^{17}$.

Très clairement, donc, le plaisir et la jouissance existent chez la femme comme chez l'homme. Le plaisir est toujours à l'origine de la production d'une semence et la jouissance est toujours accompagnée par l'excrétion du liquide séminal. La seule différence est, pour ainsi dire, qualitative et quantitative : la jouissance est beaucoup moins forte que celle de l'homme, mais elle dure plus longtemps.

C'est ainsi en vertu de la réunion de ces deux semences produites lors de l'acte sexuel, qu'est engendré l'embryon qui se développera pendant la gestation :

[...] La matrice après avoir reçu le sperme et s'être fermée, le garde en elle, puisque son orifice se resserre sous l'effet de l'humeur, et ce qui provient de l'homme et de la femme s'y mélange $(\mu i ́ \sigma \gamma \varepsilon \tau \alpha \mathrm{l})^{18}$.

L'acte sexuel et le plaisir qui l'accompagne sont donc à l'origine de la production d'une double semence qui procède de toutes les parties du corps de l'homme, comme de la femme. Dans le cadre de cette théorie, le phénomène du plaisir constitue une preuve du fait que la génération d'un nouvel individu se fait par l'union de la semence des deux géniteurs, dont le plaisir se trouve à l'origine du processus de production de leur semence. La thèse est donc là aussi fondée sur l'idée que le plaisir est le signe d'une production séminale.

Essayons de comprendre maintenant le sens de la critique qu'Aristote adresse à cette thèse. Le cœur de la réponse d'Aristote consiste à expliquer que le plaisir intense ( $\dot{\eta} \delta$ ovì $\sigma \varphi \circ \delta \rho \alpha ́$ ) qui se produit lors du rapport sexuel

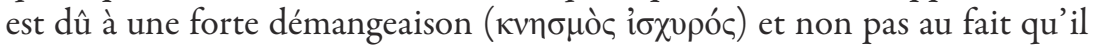
se produit dans toutes les parties du corps. Contre les tenants de la doctrine pangénétique, Aristote assure que le plaisir intense n'est pas causé par le fait que tout le corps produit de la semence, mais par le fait qu'il y a un chatouillement très vif qui se produit dans une partie spécifique du corps et qui s'éteint au moment de la jouissance.

Aristote établit ainsi un lien d'implication directe entre cette démangeaison, la production du liquide séminal, le plaisir et la jouissance. Il conclut que si la semence ne venait pas d'une seule partie, mais de toutes, l'homme devrait éprouver une série de jouissances se produisant l'une après l'autre dans toutes les parties du corps par lesquelles la semence est engendrée. En effet, si le plaisir est l'effet d'une production séminale opérée par toutes les

17. Genit. IV 1, p. 47, 11-19 (Joly 1970).

18. Genit. V 1 (Joly 1970). 
parties du corps et que la jouissance en est l'aboutissement, on devrait avoir une série de jouissances qui se produisent, les unes après les autres, dans toutes les autres parties du corps, après celle qui se produit dans l'organe sexuel - ce qui n'est évidemment pas le cas.

La réponse d'Aristote à l'argument « empirique » d'origine hippocratique se situe donc, pour ainsi dire, sur le même plan. Les données de l'expérience contredisent, selon Aristote, l'argument qui se trouve au fondement de cette thèse pangénétique. On constate par les sens que la jouissance ne se produit que dans une partie du corps : dans l'organe sexuel ${ }^{19}$. Aristote ne parle pas ici du plaisir de la femme, mais cela n'a rien d'étonnant, car son but premier est moins d'expliquer positivement ce qu'est le plaisir, que d'écarter la thèse pangénétique.

Aristote semble donc conclure que l'intense démangeaison, qui est cause de l'intense plaisir, ne se produit pas dans tout le corps et que c'est pour cela que l'intensité ne constitue pas une preuve en faveur de la pangenèse. Il semble également affirmer que la jouissance est localisée dans une seule partie, celle dont la semence est évacuée, et qu'elle constitue l'achèvement de la démangeaison en question ${ }^{20}$.

Sur la base de cette critique, on peut donc conclure que, si la démangeaison et le frottement, ainsi que le réchauffement des parties sexuelles lors de l'acte sexuel, produisent du plaisir, la jouissance constitue le sommet du plaisir et se produit instantanément, au moment où l'on parvient à la fin de l'acte. C'est à cette conclusion que poussent les lignes 723b32-724a3 du passage cité, qui concluent implicitement qu'on a une seule jouissance, qui se produit d'un seul coup, et non pas plusieurs qui se produisent, les unes après les autres, dans les diverses parties du corps.

De façon positive, ce texte nous permet également de conclure qu'Aristote, en dépit de la critique qu'il adresse à la théorie pangénétique, lui reprend deux idées : l'idée que le plaisir suit une démangeaison, due à un réchauffement et à la présence d'un corps résiduel ; l'idée que la jouissance finale, par l'évacuation du résidu, achève la démangeaison comme étant la fin d'un mouvement. Son désaccord explicite porte, en revanche, sur l'origine et

19. Cet argument confirme donc l'attitude « empirique » qu'Aristote adoptait dans ses recherches sur le rôle de la femme dans la génération. Sur cet aspect de la recherche aristotélicienne, voir Connell 2000.

20. On verra qu'Aristote ne veut pas conclure que le plaisir vient simplement du chatouillement de la partie concernée, car la production et l'expulsion du pneuma jouent un rôle en un sens plus fondamental. En ce sens, il faut considérer la critique d'Aristote comme un argument ad hominem, visant à exclure que le plaisir total implique une production globale de semence. L'argument de la répétition évoqué dans le même passage (le plaisir diminue si on éjacule plusieurs fois de suite, alors que la semence est produite à chaque fois) semble en ce sens avoir un rôle plus positif. En effet, il semble qu'il permet d'assurer le lien direct entre le plaisir et le chatouillement lui-même. 
la localisation de la démangeaison et de la jouissance. Il reste maintenant à comprendre s'il porte également sur le fait que le liquide expulsé soit nécessairement une semence. Si l'on admet cette hypothèse, on sera effectivement obligé soit d'admettre que la femme produit une semence, soit de nier l'existence d'un plaisir sexuel féminin.

\section{2. Rétention et relâchement du pneuma : contre la théorie de la double semence}

Plusieurs textes dans la suite du $G A$ confirment que le plaisir sexuel, d'après Aristote, est lié à la présence ou plutôt à l'accumulation d'un certain résidu dans les organes sexuels. Les mêmes textes confirment que cette accumulation est la source de la démangeaison et donnent des indications supplémentaires sur la nature de ce résidu. Aristote y explique notamment que l'échauffement qui accompagne la production de ce liquide rend celui-ci plus ou moins apte à féconder la matière féminine ${ }^{21}$.

À partir de l'ensemble de ces textes, on peut reconstruire de la façon suivante le phénomène du plaisir sexuel et de l'appétit qui lui est lié : un certain échauffement produit le premier changement du sang, qui produit à son tour la première démangeaison. Le premier échauffement est concomitant avec la manifestation de l'appétit sexuel, qui pousse au frottement de la partie concernée ${ }^{22}$. La démangeaison et le frottement produisent à leur tour une chaleur supplémentaire qui cause une production supplémentaire de liquide résiduel et une intensification de sa qualité. Ce corps « étranger » demeure enfermé dans les organes sexuels, jusqu'à ce qu'il se forme en lui une quantité suffisante d'un corps subtil, le pneuma, qui lui donne la puissance de sortir du corps de l'animal, en provoquant le soulagement et le plaisir instantané de la jouissance.

C'est donc cette pression progressive exercée par le corps pneumatique sur le conduit qui augmente l'appétit sexuel et pousse l'animal à continuer de frotter la partie sexuelle jusquà ce que le liquide spermatique soit expulsé. L'achèvement du plaisir coïncide donc avec l'expulsion de ce corps devenu étranger et pour ainsi dire auto-moteur ${ }^{23}$. Mais la question demeure de comprendre s'il s'agit nécessairement d'une semence qui est expulsée lors de la jouissance. On pourrait, en d'autres termes, se demander si c'est l'expulsion d'un liquide séminal, en tant que tel, à savoir d'un liquide fertile, qui

21. Voir notamment $G A$ I 6, 718 a2-4 ; I 20, 728 a9-11 ; II 4, 737 b28-738 a3. Beaucoup d'indications à ce sujet sont aussi données dans les Problèmes, notamment le 26, qui avec d'autres consacrés au même sujet (voir aussi le Problème 4) suit de façon cohérente cette explication.

22. Pour une reconstruction très claire des liens causaux entre l'appétit sexuel, le frottement et le plaisir, voir Campbell 2011, p. 354 sq.

23. Il faut en ce sens comprendre que si le liquide qui commence à être chauffé au début de l'acte sexuel n'est pas le résidu spermatique qui sera expulsé avec la jouissance, il s'agit déjà d'un liquide, pour ainsi dire, pneumatisé. 
produit la jouissance. L'ensemble des textes dans lesquels Aristote examine le phénomène du plaisir sexuel prouve que non.

En effet, l'hypothèse selon laquelle la jouissance s'identifie à l'expulsion d'un liquide fertile est infirmée par plusieurs contre-exemples. Le cas de certaines espèces, comme les poissons, dont les mâles produisent une semence qui n'est pas féconde au moment de l'éjection constitue un premier contre-exemple. Aristote explique en effet qu'en fonction de leur constitution moins chaude, les mâles de ces espèces ne sont pas capables, lors de l'accouplement, de produire une semence suffisamment chaude pour féconder la matière féminine. Dans leur cas, peut-on supposer, c'est la chaleur ambiante qui parachève l'échauffement du liquide séminal après l'expulsion et le rend fécond ${ }^{24}$.

Les jeunes garçons qui n'ont pas encore atteint l'âge d'éjaculer, les hommes stériles ou encore les vieillards, constituent un autre contre-exemple de la thèse qui voudrait que le plaisir ne se produise qu'en concomitance avec l'expulsion d'une semence. Aristote nous le dit très clairement dans un passage qui précise que ce phénomène se produit à la suite de la rétention du pneuma:

Le fait que survienne du plaisir au cours de l'union (бovovoía) ne vient pas seulement de l'émission du sperme, mais aussi du souffle ( $\pi v \varepsilon v ́ \mu \alpha \tau o \varsigma)$ au terme de la contraction duquel le sperme se répand. C'est ce qu'on voit dans le cas des enfants qui ne peuvent pas encore éjaculer mais sont presque en âge de le faire et des hommes qui ne sont pas féconds, car tous éprouvent du

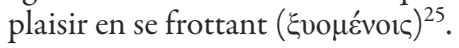

Ce n'est donc pas l'émission d'une semence qui cause le plaisir, mais la suspension et le relâchement du pneuma ou, pour être plus précis, ce n'est pas l'émission d'une semence en tant que semence qui produit le plaisir. Il est en effet clair que, pour expliquer ce phénomène, il faut pour ainsi dire remonter à une cause plus générale qui puisse expliquer aussi les cas qui posent problème : c'est la libération du pneuma et du résidu chaud produit par l'acte sexuel qui est à l'origine de la jouissance finale.

La nature du pneuma ici mentionné qu'on doit retenir et relâcher lors de l'acte sexuel n'est toutefois pas claire. On pourrait traduire $\pi v \varepsilon v \hat{\mu} \alpha$ par souffle et estimer qu'il s'agit de la respiration. Dans ce cas, il désignerait le souffle au sens ordinaire du terme et non pas directement le produit du raffinement du sang. Dans les notes de sa traduction, D. Lefebvre argumente en faveur de cette hypothèse et renvoie à $G A \mathrm{I} 6,718$ a2-4, où Aristote affirme que l'émission du liquide spermatique exige la rétention du souffle $\mathrm{e}^{26}$. Il précise aussi que

24. Voir $G A$ I 6, 718 a7-8.

25. GA I 20, 728 a9-14. Sur le plaisir sans éjaculation comme signe du fait que le plaisir ne produit pas forcément une semence, $c f .739$ a 25-26.

26. Je renvoie à la traduction commentée inédite de D. Lefebvre. Je le remercie vivement d'avoir mis à ma disposition ce travail fondamental avant sa parution. 
les cas où l'émission du résidu n'est pas produite sans la rétention du souffle, comme dans les émissions nocturnes, ne constituent pas un contre-exemple. En effet, Aristote dit simplement que l'émission nocturne du résidu, quel qu'il soit, s'effectue sans aucun effort ${ }^{27}$. Les deux passages, explique D. Lefebvre, ne sont donc pas en contradiction, si on admet qu'Aristote suggère qu'on peut retenir et relâcher le souffle, sans forcément faire d'effort.

Contre cette hypothèse, M. Campbell a suggéré que le souffle en question n'est pas premièrement ou simplement l'air dans les poumons, que l'on retiendrait avant l'expulsion, mais tout corps pneumatisé qui est élaboré lors de l'acte sexuel, qui se contracte dans les conduits et tend à être expulsé avec le corps spermatique, produit de la réélaboration finale ${ }^{28}$. Le pneuma dont la rétention et le relâchement produirait le plaisir ne serait donc ni la respiration ni le seul corps parfaitement pneumatisé, mais tout corps engagé dans le processus de pneumatisation.

Tout en se situant à deux niveaux d'explication différents, ces deux hypothèses ne sont pas forcément incompatibles. Même si, dans le texte en question, le terme pneuma semble plus renvoyer au souffle au sens ordinaire, dans d'autres contextes du même type, le terme renvoie clairement au corps subtil produit par l'échauffement du sang, de sorte que les deux explications puissent être combinées. En ce sens, on peut supposer que c'est aussi bien la production pneumatique que la rétention de la respiration, lors de l'expulsion du liquide spermatique, qui causent d'abord le plaisir, puis la jouissance. Le pneuma, dans les deux sens du mot (mais non pas dans les mêmes textes), serait donc à l'origine du phénomène du plaisir : d'une part parce qu'il est impliqué, en tant que produit du raffinement du sang, dans la production du liquide spermatique, d'autre part parce que l'émission de ce dernier est précédée par la rétention du souffle au sens ordinaire du mot.

Le plaisir sexuel serait donc l'achèvement de la démangeaison provoquée par l'appétit et intensifiée par l'accumulation, dans les organes sexuels, du corps pneumatique généré par la chaleur ${ }^{29}$. Mais est-ce qu'Aristote envisage d'utiliser ce même paradigme dans le cas des femelles ? Il semblerait que oui. Non pas justement parce que leur plaisir suit la production d'une semence, mais parce qu'il résulte de la production, de l'accumulation et de l'éjection d'un corps pneumatique. Aristote ne le dit pas explicitement, mais on peut le déduire des passages dans lesquels il affirme que les femelles non seulement émettent elles aussi un liquide lors de la copulation, quoique non séminal, mais qu'elles l'émettent au moment où elles éprouvent du plaisir :

27. GA II 4, $737 \mathrm{~b} 35-738 \mathrm{a} 1$.

28. Campbell 2011, p. 357.

29. Le « corps pneumatique » serait en ce sens le pneuma qui se produit dans les liquides chauffés. 
Certains croient que la femelle apporte du sperme au cours de l'union

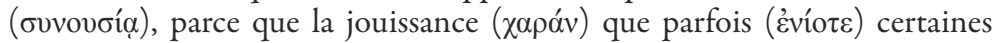
éprouvent est comparable $(\pi \alpha \rho \alpha \pi \lambda \eta \sigma i ́ \alpha v)$ à celle des mâles et que se produit en même temps une sécrétion humide, mais cette humidité n'est pas de nature spermatique; c'est une humidité de cette région du corps propre à chaque femelle ${ }^{30}$. (Trad. D. Lefebvre légèrement modifiée.)

Ce texte confirme donc qu'il y a d'après Aristote une jouissance féminine et une émission de liquide qui l'accompagne, même si ce liquide n'est pas fécond. Aristote semble pourtant poser deux limitations à l'extension du modèle au cas de la femelle : d'une part, le plaisir que la femelle éprouve est « comparable » à celui des mâles, mais non pas identique; d'autre part, il ne se produit pas nécessairement, mais « parfois ».

Le plaisir féminin n'est donc pas exactement le même que celui de l'homme et, contrairement à celui-ci, il ne se produit pas systématiquement. Le caractère non systématique du plaisir féminin constitue alors une preuve suffisante pour invalider l'argument des tenants de la double semence qui, comme l'argument du plaisir total, procède par signe de la constatation d'un plaisir sexuel à l'affirmation de la production d'une semence.

Cette même double limitation intervient aussi dans un autre passage dans lequel Aristote attaque à nouveau les tenants de la thèse de la double semence :

Mais un signe ( mâle et que la génération ne vient pas du mélange des deux, comme certains l'affirment, c'est le fait que souvent ( $\pi$ o $\lambda \lambda \alpha$ ákıৎ) la femelle conçoit sans avoir eu de plaisir au cours de l'union. Mais quand, au contraire, elle n'en a pas eu moins et que mâle et femelle ont fait la course ensemble, la génération n'a pas lieu, si la sécrétion humide de ce qu'on appelle les menstrues n'est pas proportionnée ${ }^{31}$.

Dans ce texte la limitation n'intervient que pour définir la fréquence avec laquelle le plaisir féminin se produit. Ce dernier n'est pas moindre que celui des mâles, mais il ne se produit pas aussi souvent. Là aussi la limitation est considérée comme un contre-argument suffisant à invalider l'inférence qui voudrait établir la capacité de produire une semence à partir de l'existence d'un plaisir sexuel chez les femmes. On constate, en effet, qu'il peut y avoir procréation là où il n'y a pas eu de plaisir chez la femme.

L'existence d'une sécrétion humide, assure ainsi Aristote, et d'un plaisir chez les femmes n'explique pas à elle seule la procréation, car la production de cette humidité n'implique pas celle de l'élément avec lequel la femme contribue à la génération : le sang menstruel. La femme peut avoir du plaisir, sans forcément engendrer. Tout le long du livre I, en effet, Aristote s'efforce 
de montrer que la femme ne contribue pas à la formation de l'embryon par ce résidu spermatique, mais par le premier type de liquide, à savoir le sang menstruel ${ }^{32}$.

La production d'un liquide spermatique n'est donc pas une cause suffisante pour qu'il y ait génération. Preuve en est, affirme Aristote dans un autre passage, le fait que le lieu où se produit ce plaisir n'est pas celui dans lequel se produit le liquide :

Un signe ( $\sigma \eta \mu \varepsilon i ̂ v)$ de ce que la femelle n'émet pas de sperme est aussi le fait que, durant l'accouplement, le toucher produit chez elles du plaisir dans la même région que les mâles, sans que ce soit de là que cette sécrétion humide est émise ${ }^{33}$.

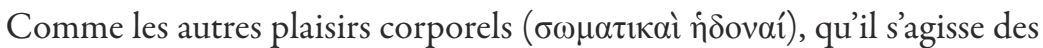
plaisirs concernant la nutrition ou des actes sexuels ${ }^{34}$, le plaisir sexuel féminin est lié au sens du toucher. Il intéresse donc une partie précise dans laquelle se produisent le contact et le chatouillement qui est à son origine. Cependant, argumente Aristote, la sécrétion humide qui est produite lors de l'acte sexuel n'est pas engendrée, comme c'est le cas de l'homme, par la partie dans laquelle on éprouve le plaisiri ${ }^{35}$, mais elle procède de l'utérus. En effet, comme Aristote l'explique au livre $\mathrm{II}^{36}$, la sécrétion spermatique qui se produit parfois chez les femmes est produite dans la partie antérieure de l'utérus. Le fait que le plaisir ne soit pas logé dans la partie qui produit le liquide spermatique constitue donc une preuve supplémentaire, appelée explicitement « signe » ( $\sigma \eta \mu \varepsilon \hat{\imath o v})$, du fait que le plaisir chez la femme n'est pas accompagné par la production d'une semence.

Sur cette donnée empirique repose également un autre argument qu'Aristote utilise pour montrer que le liquide spermatique de la femme n'est pas une semence. En effet, si ce résidu était un liquide séminal, il faudrait supposer qu'il est attiré à l'intérieur de l'utérus juste après en avoir été expulsé. Dans ce cas, cependant, on serait obligé d'admettre que la nature a fait quelque chose en vain ${ }^{37}$.

Le liquide non fertile, tout comme le plaisir qui accompagne son expulsion, n'est donc pas suffisant pour expliquer la génération, mais il n'est pas non plus nécessaire, puisque, comme on l'a vu, la femelle peut engendrer sans plaisir. Le plaisir féminin, dû à l'évacuation du liquide sexuel, existe mais à la différence de celui du mâle, il n'est ni nécessaire ni suffisant pour la

32. Sur ce point, voir Cerami 2015 p. 129-147, ainsi que la bibliographie citée.

33. GA I 20, 728 a31-34.

34. Cf. notamment Aristote, EN VII 6, 1147 b25-28.

35. Aristote ne le dit pas, mais il s'agit sans doute du clitoris, voir à ce propos Dean-Jones 1992.

36. GA II 4, 739 a10-b20.

37. $G A$ II 4, 739 b16-20. Je remercie l'auteur de la relecture anonyme pour avoir attiré mon attention sur cet argument supplémentaire. 
génération. Peut-on dès lors affirmer que le phénomène du plaisir n’a, chez les femelles en général et chez la femme en particulier, aucune véritable raison d'être ? Et que le liquide associé est comparable à d'autres liquides non utiles qui se produisent en excès, comme le flegme, auxquels Aristote ne semble accorder qu'une nécessité purement matérielle?

\section{3. Plaisir et téléologie :}

explication «par la nécessité », explication « en vue du mieux»

Aristote ne s'exprime pas très clairement sur la raison d'être du plaisir sexuel féminin, mais un passage du livre II nous fournit des éléments essentiels. Après avoir confirmé que l'humeur produite chez les femmes au moment de la jouissance ne joue aucun rôle dans la conception et que cette dernière peut avoir lieu sans qu'il se produise aucun plaisir féminin, Aristote précise au chap. 4 que la jouissance féminine accompagne toutefois « habituellement $\gg$ la procréation :

Donc, sans l'émission du mâle durant l'accouplement et sans le résidu menstruel, qu'il sorte au dehors ou qu'il y en ait suffisamment à l'intérieur, la conception est impossible; en tout cas, elle a lieu même sans que survienne le

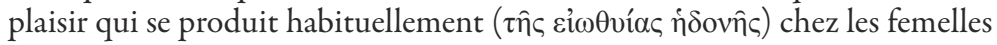
dans ce type de relation, pourvu en tout cas que la région soit excitée et que l'utérus soit descendu vers le bas ${ }^{38}$.

Le plaisir sexuel féminin, la jouissance et la production d'un résidu, bien qu'ils ne soient pas nécessaires à la procréation, c'est-à-dire en vue de la génération, l'accompagnent « le plus souvent ». Pour cela même, à la différence du plaisir masculin, la jouissance féminine n'est pas un phénomène nécessaire à la génération. En effet, le plaisir sexuel de l'homme est absolument nécessaire, car le canal ne prend la bonne position qu'au moment où l'homme a du plaisir et quand ce plaisir touche à sa fin. Dans les individus mâles achevés, en effet, le plaisir a la fonction nécessaire de positionner de la bonne façon le canal spermatique et de permettre l'émission de la semence. Chez la femme, en revanche, le plaisir n'est pas nécessaire, parce que l'utérus, lorsqu'il est bien chauffé, peut attirer de lui-même la semence.

On peut donc conclure que le plaisir féminin n'est pas un phénomène qui existe en vertu de la survie de l'animal ou de sa génération. En effet, il n'est pas comparable aux phénomènes qui se produisent en vue d'une finalité absolue, tels que la production du sang ou de la semence. Il ne relève toutefois ni d'une causalité purement mécanique, ni de l'ordre de l'accidentel. Cette conclusion est

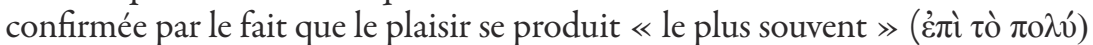
et qu'il contribue en quelque sorte à la procréation. Aristote explique, en effet, que le plaisir sexuel et la formation spermatique qui l'accompagne produisent le mouvement du col de l'utérus qui permet à la semence d'y pénétrer : 


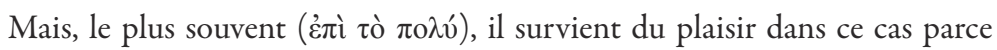
que, quand l'excrétion, durant laquelle naît habituellement le plaisir chez les mâles comme chez les femelles, s'est produite, le col de l'utérus ne s'est pas refermé ; et quand il en va ainsi, la voie est aussi plus dégagée pour le sperme du mâle ${ }^{39}$.

Le plaisir féminin ne se produit donc pas toujours, mais le plus souvent. C'est pour cette même raison qu'on peut le définir comme la condition naturelle à laquelle l'individu est disposé et dans laquelle par nature il doit se trouver. Mais quel est donc, de ce point de vue, le type de causalité qui lui est propre ? Ce que je voudrais suggérer, c'est que le plaisir féminin doit être considéré comme l'un des phénomènes biologiques qu'Aristote définit comme existant non pas nécessairement, mais « en vue du bien » (

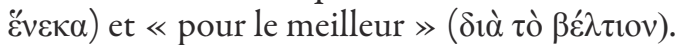

Avant même de comprendre en quel sens le plaisir féminin serait « en vue du bien » et « pour le meilleur », considérons quelques autres phénomènes biologiques appartenant à cette même classe. Cela permettra de mieux saisir la nature propre du plaisir féminin et de clarifier d'une façon générale le type de causalité en jeu.

La distinction entre ces deux types d'explication, par la nécessité et par le meilleur, est maintes fois évoquée dans le $G A$ et plus généralement dans l'ensemble du corpus biologique ${ }^{40}$. Dans tous ces textes, l'explication « en vue du bien » intervient à chaque fois que la nature se trouve devoir faire face à un obstacle, pour ainsi dire, connaturel, qu'il s'agisse d'un obstacle dû à un excès ou à un défaut. Aristote explique qu'en agissant en vue du meilleur, la nature contourne l'obstacle ou, comme Aristote le dit en $G A$ III 4 (755 a31),

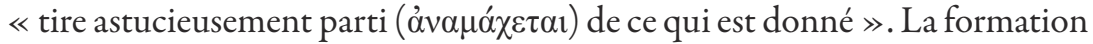
des menstrues constitue un exemple emblématique de ce type de finalité :

Pour les raisons que nous venons de donner, c'est donc par nécessité (’̊ $\xi$

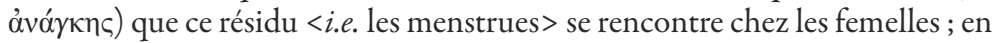
effet, comme la nature est impuissante à opérer la coction, il est nécessaire que se forme non seulement un résidu de la nourriture inutile mais aussi un résidu dans les vaisseaux et qu'en s'accroissant le long des vaisseaux les plus fins, il

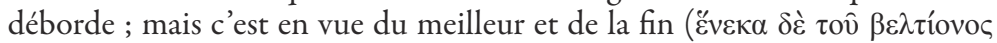

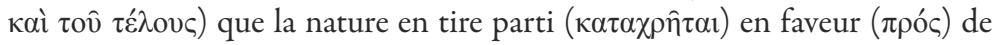
cette région du corps qui est pour la génération afin que soit engendré un autre être du type qu'il doit être : en effet, il existe à partir de là du moins en puissance, en étant tel que le corps dont il est la sécrétion ${ }^{41}$.

Ce passage montre bien qu'un même phénomène, comme la formation des menstrues, peut être considéré comme la conséquence d'une nécessité

39. $G A$ II 4,739 a31-35.

40. Le principe du meilleur est décrit en PA III 2, 663 b22-24; $c f$. Aristote, $G A$ II 4, 738 a-b4 ; III 4, 755 a24 sq. ; IV 8, 766 b31 sq. ; V 8, 798 a8-b5.

41. $G A$ II 4,738 a33-b4. 
matérielle, à savoir l'incapacité connaturelle de l'organisme féminin à opérer la coction, mais aussi comme un phénomène téléologiquement orienté. La causalité finale qui caractérise ce phénomène n'est toutefois pas de même nature que celle qui gouverne la production de la semence du mâle. La production de la semence fait partie des fonctions nécessaires de l'espèce et constitue, pour cela même, une propriété constitutive de l'essence. Car cette production est le moyen direct de réaliser la fonction propre à la forme spécifique achevée, à savoir la génération d'un autre être de même type. Dans le cas des menstrues, nous dit en revanche Aristote, la nature « tire parti » de la surabondance d'un résidu non cuit et elle en fait quelque chose d'utile « pour autre chose ».

À juste titre, dès lors, Aristote parle de finalité « en vue du meilleur ». En effet, le résidu non cuit n'a pas par soi et en tant que tel une finalité intrinsèque; l'existence de ce résidu n'est pas, pour le dire autrement, un bien absolu, mais il le devient du moment qu'il est investi d'une nouvelle fonction, celle d'être en puissance le nouvel individu engendré. La « condition d'optimalité $\gg{ }^{42}$ propre à l'existence des menstrues serait en ce sens non pas absolue, mais « relative à autre chose », car elle est « en faveur de la région du corps qui est pour la génération », à savoir l'utérus. Mais le fait d'être « en faveur de cette partie » est également pour les menstrues mieux que de rester ce qu'elles sont, à savoir un simple résidu. Comme dans le cas des esclaves $^{43}$, mais on pourrait le dire aussi de la femme en général, les menstrues sont donc tournées vers quelque chose qui est un bien pour elles aussi. En effet, le « meilleur » est, pris absolument, la fonction primaire des organes reproductifs ; mais dans le cas des menstrues elles-mêmes, c'est le fait d'y contribuer ${ }^{44}$.

Par ailleurs, il faut souligner que la surabondance de sang dans les espèces dans lesquelles il y a une division entre mâle et femelle découle de la surabondance de chaleur qui leur est propre. En effet, la séparation du principe mâle implique nécessairement une surabondance de chaleur. En ce sens, l'existence des menstrues est une conséquence de l'excellence des espèces dans lesquelles le principe mâle est séparé du principe femelle.

42. J'appelle « condition d'optimalité » l'état que la finalité en vue du meilleur réalise.

43. Aristote explique en effet en Pol. I 5 que, pour certains individus de l'espèce humaine, le fait d'être esclaves constitue une condition d'optimalité, dans la mesure où cela leur permet de contribuer à un bien absolu qui est celui réalisé par leur maître. C'est dans le même sens, en effet, qu'il faut entendre que le fait d'être gouverné, c'est pour eux quelque chose de meilleur.

44. On pourrait en ce sens affirmer que la condition d'optimalité est à la fois subjective, dans la mesure où la nouvelle fonction dont le sujet en question (les menstrues, par exemple) est investi est un « mieux » pour lui, mais aussi objective, car la fonction en faveur de laquelle le sujet est tourné (la fonction reproductive) est en soi un bien absolu. En ce sens, pour le sujet (toujours les menstrues, par exemple) la condition d'optimalité deviendrait absolue. Je vais revenir sur ce point. 
Comme le suggère Aristote, en effet, c'est cette séparation qui détermine le degré maximal de noblesse des animaux dans lesquels les deux principes formel et matériel sont séparés ${ }^{45}$.

Un autre phénomène biologique relève de ce même type de finalité et clarifie la valeur double de la condition d'optimalité. C'est le cas des reins pour les animaux qui en possèdent. Aristote explique en effet que les reins appartiennent aux animaux qui en ont, non pas « par nécessité », mais « en vue du bien et du beau » :

Les reins appartiennent à ceux qui en ont, non pas nécessairement ( $\dot{\varepsilon} \xi$

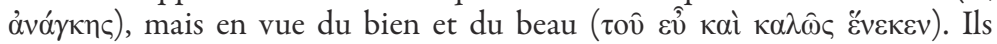

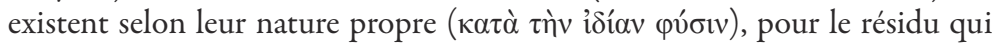
s'accumule dans la vessie chez les animaux chez lesquels un tel dépôt se trouve plus abondant, afin que la vessie remplisse mieux sa fonction (ö $\pi \omega \varsigma$

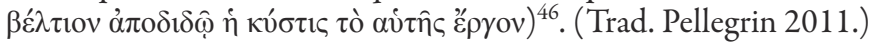

Les reins ont une utilité, pour ainsi dire, dérivée ou relative, dans la mesure où ils appartiennent aux animaux qui les possèdent afin qu'une autre partie, à savoir la vessie, remplisse mieux sa fonction. En effet, les animaux qui possèdent une vessie ne possèdent pas tous des reins. Seuls ceux qui possèdent des poumons sanguins les ont. L'existence des reins devient effectivement utile à cause de l'excès de liquide qui se produit dans cette classe d'animaux qui, du fait de leur nature chaude, ont besoin d'assimiler beaucoup de liquide ${ }^{47}$. Cependant - assure Aristote - les animaux qui possèdent une vessie ont, dans la plupart des cas, des reins ${ }^{48}$. Là aussi donc la surabondance de liquide est la conséquence indirecte de l'excellence de l'espèce concernée.

Revenons maintenant au cas du plaisir féminin. L'explication qu'Aristote en a formulée montre qu'il rentre lui aussi dans cette même classe de phénomènes biologiques. Ce plaisir ne peut appartenir à la classe des phénomènes biologiques qui découlent d'une nécessité absolue, car, comme on l'a vu, il n'est pas nécessaire à la réalisation de la fonction propre au vivant considéré, à savoir le fait de reproduire un être semblable. Il peut en revanche s'expliquer par la seconde de ces deux raisons, à savoir le fait d'être en vue du meilleur. En effet, bien que l'explication d'Aristote ait assurément un aspect mécanique, elle montre aussi que ce plaisir possède véritablement une certaine fin, à savoir le fait de faciliter la procréation, en favorisant chez la femme l'ouverture du canal sexuel par lequel passe le sperme masculin.

Du point de vue de la génération, le plaisir féminin peut donc ne pas exister, mais s'il existe dans la plupart des cas, c'est qu'il permet d'optimiser la fonction de l'organe qui réalise directement la fonction primaire en question,

45. GA II 1,732 a1-11.

46. $P A$ III 7, 670 b23-27.

47. $P A$ III 8,670 b32-671 a9.

48. $P A$ III 9, 671 a26-671 b3 ; GA IV 4, 771 a2-6. 
à savoir l'organe reproductif féminin. En effet, comme Aristote l'a expliqué, il permet au col de l'utérus de s'ouvrir, en rendant la voie « plus dégagée pour le sperme du mâle ». Dans le cadre de cette explication, il est donc clair que le liquide spermatique féminin n'est ni un simple lubrifiant, ni un résidu visant à attirer à l'intérieur de l'utérus le sperme du mâle ${ }^{49}$. Aristote explique en effet clairement que cette fonction est propre à l'utérus en raison de sa chaleur, tandis que la fonction propre au plaisir sexuel est, d'une façon analogue à ce qui se passe pour le mâle, de bien orienter le canal qui conduit à l'utérus, afin de faciliter l'entrée de la semence.

Dans le cas du plaisir féminin, donc, comme dans le cas des menstrues, la nature met quelque chose, qui n'est pas en tant que tel un bien en soi, au profit de ce qui est meilleur. L'existence de ces phénomènes est définie comme non nécessaire, mais expliquée en raison d'un état d'optimalité des animaux chez qui ils se manifestent. Ces phénomènes ne se produisent pas en fonction de la survie de l'animal, mais pour améliorer les fonctions d'une autre partie, qui existe, elle, en vertu d'une nécessité au sens absolu. Mais est-ce que cela implique une forme de finalité de niveau inférieur à celle de la nécessité absolue ? En conclusion de cette partie de mon étude, je voudrais suggérer que non.

Dans les trente dernières années, la distinction entre ces deux types d'explication, par la nécessité et par le meilleur, a attiré l'attention croissante des spécialistes ${ }^{50}$ qui, en soulignant le caractère téléologique de l'explication par le meilleur, ont pointé le rôle crucial que cette dernière joue dans la biologie aristotélicienne. Dans la plupart de ces études, de façon plus ou moins marquée, on a insisté sur le fait que la distinction entre l'explication par la nécessité et l'explication en vue du meilleur peut se lire à la lumière de la distinction entre propriétés formalo-essentielles et propriétés provenant de la matière. L'explication par la nécessité se rapprocherait asymptotiquement de la forme-essence de la substance vivante. L'explication par le meilleur s'en éloignerait progressivement, jusqu’à être ultimement résorbée par la nécessité procédant de la matière, notamment dans le cas de certains phénomènes particuliers comme, par exemple, la présence des cornes dans les cerfs ${ }^{51}$.

L'un des plus grands mérites de cette littérature a été en ce sens de mettre en lumière l'existence de plusieurs niveaux de finalité au sein des phénomènes téléologiquement orientés. Le fait toutefois d'utiliser de façon trop stricte

49. Ce type d'explication a été proposé dans Campese, Manuli \& Sissa 1983, p. 125.

50. Sur l'importance de l'explication par le meilleur, voir les travaux pionniers de Balme 1987 ; Gotthelf 1987 ; Lennox 1997a et 1997b ; Code 1997. Pour plus d'exemples et pour une discussion plus générale sur la distinction entre ce qui est nécessaire et ce qui est en vue du mieux, Gotthelf 1989 ; Pellegrin 1986, 1990 et 2002. Pour une mise en contexte « métaphysique » de cette notion, voir Rashed 2002.

51. Aristote, $P A$ III 2, 663 b22-29. 
la distinction entre cause formelle et cause matérielle pour interpréter l'existence de ces différents niveaux ne me paraît pas tout à fait approprié52. D'un côté, en effet, on pourrait croire qu'il est possible de distinguer en acte, au sein des vivants, entre une forme, dont découleraient les propriétés nécessaires à la survie de la substance, et une matière, dont ne résulteraient que les excès et les défauts. D’un autre côté, le fait de rabattre les différents niveaux de finalité sur la différence entre forme et matière masque l'un des enjeux les plus cruciaux de cette doctrine : l'horizon d'application de la condition d'optimalité.

Admettre que la matière fournit, dans le cas de la téléologie « dérivée », des « possibilités supplémentaires » par rapport à celles auxquelles la forme pourvoit impliquerait que dans le cas de la téléologie « directe » elle ne joue aucun rôle. Ce qui est nié par tous les passages dans lesquels Aristote assure qu'un même phénomène peut être expliqué à la fois comme nécessaire et en vue de la préservation du vivant ${ }^{53}$. En un sens, à tout moment, pendant la génération et jusqu’à la mort de l'être animé, les propriétés venant des composants matériels sont en interaction avec les propriétés assurées par la forme. Les phénomènes liés aux fonctions primaires, comme les excès et les résidus, sont le produit de cette interaction. Dans le cas des résidus, en effet, la nature « permet » leur production non pas après coup, mais ab origine ou, pour le dire autrement, potentiellement dès le début, dans le but de les intégrer dans la phase de recyclage de la matière utilisée pour les fonctions primaires. En d'autres termes, les phénomènes en vue du mieux sont déjà inscrits dans la forme, non pas à titre premier, mais comme conséquences dérivées de l'essence ${ }^{54}$.

Par ailleurs, il faut également préciser en quel sens ces phénomènes sont non nécessaires ; c'est cela qui permet de déterminer l'horizon d'application de la condition d'optimalité. Affirmer la non-nécessité des phénomènes

52. En dépit de la clarté et du caractère synthétique de son analyse, cette difficulté me paraît particulièrement évidente dans la distinction proposée par M. Leunissen entre « finalité primaire » et « finalité secondaire » ( $c f$. Leunissen 2010, p. 81-111). La « finalité primaire » serait liée à la nécessité dite hypothétique, la « finalité secondaire » à la « nécessité matérielle ». Cette dernière, explique Leunissen, en interaction avec les actions réalisées en vertu de la forme, offrirait à la nature des possibilités supplémentaires (« extra possibilities ») qui nétaient pas fournies dans l'âme que l'animal en question possède (ibid. p. 111). Elle conclut en ce sens que, d'après son interprétation, à la différence de la majorité des autres, la nécessité matérielle joue un rôle véritablement positif (p. 99).

53. Mon interprétation se rapproche davantage de celle défendue dans Cooper 1987 (cf. notamment p. 255-259) et Lennox 1987.

54. On peut en ce sens envisager d'expliquer de la même façon d'autres phénomènes qui peuvent être considérés comme des conséquences encore plus éloignées de la finalité directe. Dans ce cas-là, il n'y aurait rien dans les organismes vivants qui serait au sens strict le produit d'une simple nécessité matérielle. Tout serait l'effet «plus ou moins éloigné » de la réalisation de la forme achevée. Je me réserve de revenir sur cette question dans une étude à part. 
« pour le meilleur » ne revient pas à admettre que l'animal, considéré comme membre de son espèce, peut survivre sans que ces phénomènes se produisent parce que ces derniers contribuent à des fonctions non essentielles ${ }^{55}$. Cela ne revient pas non plus à affirmer simplement que les phénomènes en question, sans être nécessaires à la survie de l'individu, permettent de mieux faire fonctionner des parties qui fonctionneraient même sans eux ${ }^{56}$. Je voudrais en effet suggérer que les phénomènes en vue du mieux sont non nécessaires dans la mesure où ils permettent de fixer, au sein même de l'espèce, le dernier niveau d'accomplissement formel auquel les membres de l'espèce peuvent parvenir ou pas. C'est cette condition qu'exprime Aristote, lorsqu'il affirme que les phénomènes en vue du bien contribuent au «bien être » et non simplement au «bien » de l'animal. L'individu chez qui ces phénomènes ne se produisent pas demeure un membre de la classe en question, c'est-à-dire un membre de son espèce. Toutefois, il ne l'est pas au même titre que l'individu chez qui ces phénomènes se produisent, car il n'a pas atteint son plein et total accomplissement. Au niveau spécifique, la condition d'optimalité permet donc de définir l'excellence vers laquelle l'espèce même est orientée.

Ce même raisonnement doit s’étendre également au niveau générique. La condition d'optimalité, en effet, ne se comprend pas simplement à l'intérieur de l'espèce, mais doit être évaluée au niveau de la classe à laquelle l'espèce appartient. En d'autres termes, le phénomène en vue du bien ne se comprend comme meilleur, pour l'espèce dans laquelle il se produit, que lorsque l'on prend en compte les autres espèces de la même classe dans lesquelles le phénomène ne se produit pas $^{57}$. Le fait de posséder une fonction qui n'est pas une nécessité absolue, mais un bien relatif permet en ce sens de classer les différentes espèces d'une même classe, en les considérant comme plus ou moins parfaites ${ }^{58}$.

Au niveau spécifique, comme au niveau générique, la condition d'optimalité n'est donc pas le signe d'une défaillance de la forme, sur laquelle la matière l'emporterait au moins en partie, elle est le signe du statut supérieur

\section{Leunissen 2010, p. 90.}

56. Gotthelf 1989, p. 122.

57. L'existence de la femelle doit être interprétée dans le même sens. Je résiste donc aux lectures qui font de l'existence de celle-ci le résultat d'une téléologie manquée, dans laquelle la cause finale serait « vaincue » par la cause matérielle (cette idée a été défendue par Nielsen 2008 qui définit l'explication par le meilleur comme une «proto-téléologie » [p. 379]), autant quà celles qui en font le produit d'une nécessité purement matérielle (Henry 2007). Mon approche va plus dans le sens de la lecture proposée par Charlotte Witt 1998 ; $c f$. aussi Deslauriers 1998 qui défend l'idée que la femme est le signe de l'existence d'une échelle de perfection à l'intérieur de l'espèce. J'estime toutefois que cette « perfection de niveau inférieur » doit se comprendre comme découlant indirectement de la forme spécifique et non simplement de la nécessité matérielle.

58. Sur le classement des espèces en fonction des formes qui leur correspondent, voir Cerami à paraître. 
de l'individu, de l'espèce ou de la classe générique qui la réalisent. En intégrant dans ce même schéma le plaisir sexuel féminin, on peut ainsi conclure que celui-ci n'est pas simplement une autre ruse de la nature qui opère en vue de la survie de l'espèce, mais la condition d'optimalité des individus chez qui il se produit : pour tous les individus femelles, comme pour les espèces dans lesquelles on a une distinction entre mâle et femelle, le plaisir sexuel féminin est la condition qui permet d'assurer non pas la préservation, mais le plus complet et total accomplissement de leur être.

\section{Averroès contre Galien sur la finalité du plaisir}

La question de la compréhension de la nature et de l'origine du plaisir féminin, et celle de l'explication de la fonction de la production concomitante d'un liquide spermatique ont été reprises par la tradition postérieure à Aristote, soit pour simplement signaler la difficulté, soit pour l'intégrer dans une explication plus générale des fonctions génératives animales. L'étude du débat qu'Averroès a engagé contre Galien montre qu'on trouve dans cette tradition les deux mêmes options qu'on a signalées : l'explication par la nécessité et celle en vue du mieux.

Dans la tradition péripatéticienne, Théophraste a été le premier à pointer la difficulté posée par l'émission d'un résidu féminin non séminal dans le système téléologique d'Aristote. Dans le dernier chapitre de sa Métaphysique, il ne parle pas du plaisir féminin, mais il semble inscrire l'émission du liquide spermatique féminin au nombre des phénomènes qui pourraient remettre en question le principe que la nature ne fait rien en vain. Sans préciser la relation que cette émission entretient avec le plaisir féminin, il affirme non seulement que cette dernière ne semble pas être en vue de quelque chose, mais qu'elle semble être vaine :

[...] En outre chez les animaux eux-mêmes, certains <phénomènes> sont comme vains, par exemple les mamelles pour les mâles et l'émission pour les femelles, s' il est vrai qu'elle ne contribue en rien ${ }^{59}$. (trad. A. Laks-G.W. Most.)

Les spécialistes hésitent sur le type d'émission à laquelle Théophraste fait allusion dans ce passage ${ }^{60}$. La plupart remarquent qu'il s'agit sans doute du liquide menstruel. À la lumière des difficultés qu’on a pointées, il semble toutefois plus probable que l'allusion concerne l'émission qui se produit, chez les femmes, au moment du rapport sexuel ${ }^{61}$. En effet, il est vrai que les menstrues sont un résidu dû à un excès, dont la rétention serait nuisible ; cependant, comme on l'a vu, leur production est essentiellement liée à la génération et elle ne pourrait pas être définie comme complètement inutile.

59. Théophraste, Métaphysique, 10 b7-9.

60. Voir à ce propos Laks \& Most 1993, p. 78, n. 14.

61. Aristote, GA I 19, 20, 727b36, II 4, 739 a37-b1. 
Théophraste ne dit pas explicitement si le résidu féminin constitue pour lui une véritable exception au principe selon lequel la nature ne fait rien en vain et donc à la téléologie aristotélicienne. Il se contente de soulever l'aporie et d'assimiler la production de ce liquide à l'existence des mamelles chez les hommes ou encore à la génération des cornes des cerfs qui, selon Aristote lui-même, sont non seulement inutiles pour les cerfs, mais aussi nuisibles ${ }^{62}$. Sans se prononcer sur la date de rédaction de la Métaphysique de Théophraste, qui est encore objet de débat auprès des spécialistes ${ }^{63}$, on peut ainsi signaler qu'au sein même du peripatos la production d'un résidu féminin était considérée comme problématique.

\section{1. Galien sur l'anatomie du plaisir}

La question de la nature du plaisir sexuel de la femme et de la fonction du liquide spermatique produit simultanément a été ensuite reprise par Galien qui l'a réinscrite dans le cadre de sa propre théorie générative ${ }^{64}$. S'inspirant en dernière instance des théories hippocratiques, mais appuyant aussi ses conclusions sur ses études anatomiques, Galien affirme à plusieurs reprises dans son corpus que le plaisir sexuel est, chez la femme comme chez l'homme, associé à la production d'une semence. Du fait que les femmes aussi ont du plaisir, il infère ainsi leur contribution à la procréation par un liquide spermatique fertile. L'originalité de Galien consiste alors à affirmer que l'équivalence entre les deux sexes et leur plaisir n'est pas simplement au niveau de la fonction, mais de la structure. Leur plaisir sexuel découle en effet de leurs organes reproductifs absolument analogues.

Galien aborde la question du plaisir féminin et du liquide spermatique qui y est associé dans son $D e$ Semine ${ }^{65}$. Sa solution est intégrée dans une étude plus vaste sur la fonction de chacun des deux sexes dans la reproduction. En effet, au début du traité, Galien affirme vouloir expliquer « l'utilité »

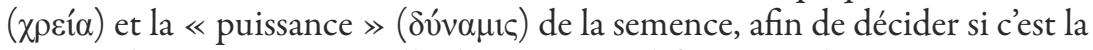
théorie d'Hippocrate ou celle d'Aristote qu'il faut considérer comme vraie. Il consacre à la semence féminine le livre II du traitéé ${ }^{66}$. Il affirme au chap. 4

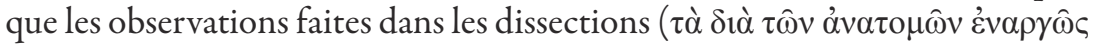
( $\alpha$ vó $\mu \varepsilon v \alpha$ ) prouvent qu'au niveau anatomique de leurs organes reproductifs les deux sexes ont une nature absolument analogue ${ }^{67}$. Les deux en effet possèdent des testicules capables de produire une semence, dont la production et

62. Aristote, $P A$ III 2.

63. Pour un état de la question, voir Gutas 2010, p. 3-9.

64. Sur la théorie reproductive de Galien, voir Accattino 1994. Voir aussi Preus 1977 ; Boylan 1984 et 1986.

65. Galien, De semine (De Lacy 1992 = Kühn IV, 512-652).

66. Galien, De semine, I 1 (De Lacy 1992 p. 64 = Kühn IV, 512).

67. Galien, De semine, II 1 (De Lacy 1992 p. 145 = Kühn IV, 593). 
l'expulsion produisent la jouissance sexuelle : il s'agit chez les hommes des testicules et chez les femmes des ovaires ${ }^{68}$, ces derniers ne se distinguant des testicules masculins que par leur taille et leur position.

Dans le même chapitre, Galien rétorque à Aristote, mais surtout à son contemporain Athénée - qui affirmait que les parties spermatiques des femmes ne sont pas fonctionnelles - qu'il ne faut pas nier l'existence d'une semence féminine sous prétexte qu'on n'en saisit pas la cause. En retournant contre Aristote le principe qu'il avait lui-même posé au fondement de sa recherche ${ }^{69}$, Galien affirme que l'existence de ce qui apparaît clairement ne doit pas être remise en question sur la base d'un simple raisonnement

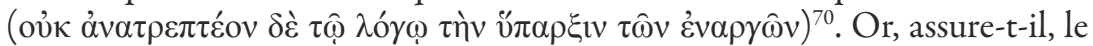
fait qu'une semence féminine existe est quelque chose que les sens rendent manifeste. Galien conclut ainsi que si le plaisir des femmes et leurs testicules n'avaient pas de véritable fonction, la nature aurait fait quelque chose en vain dans la mesure où ces derniers seraient inutiles ${ }^{71}$. Le plaisir sexuel, explique en revanche Galien, a bien une double finalité, car la production de la semence qui l'accompagne non seulement sert à produire le désir de la copulation, mais fournit ultimement à l'embryon l'une des membranes qui l'entoure : l'allantoïde.

Dans ce cadre théorique, l'existence de la femelle, caractérisée par sa nature moins chaude, de même que celle de son plaisir sexuel, sont conçus moins comme une ruse de la nature que comme une nécessité. En effet, Galien explique que le peu de chaleur qui caractérise la femme fait que ses parties spermatiques et sa semence restent à l'intérieur de son corps, afin de fournir à l'embryon qui se développe dans son utérus cette membrane, ainsi que la nourriture nécessaire à son évolution. De ce point de vue, peut-on conclure, le plaisir sexuel féminin a d'après Galien une nécessité véritablement téléologique : il existe directement en vue de la reproduction animale et de la perpétuation de l'espèce.

Cette même explication du plaisir féminin et le caractère finaliste de son existence sont affirmés et précisés dans le De Usu Partium. Au chap. 2 du livre XIV, Galien explique que le plaisir sexuel est une « puissance »

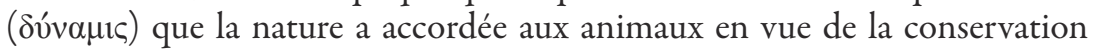
de l'espèce :

68. L'existence de cet organe était, on le sait, inconnue d'Aristote.

69. Aristote, $G A$ III 10, 760 b27-33 : « Se fier à la sensation plutôt qu'aux raisonnements, et aux raisonnements si ce qu'ils montrent est en accord avec les phénomènes ».

70. Galien, De semine, II 4, 12 (De Lacy 1992, p. 172, 12 = Kühn IV, 620, 6).

71. Toujours dans le même chapitre, Galien affirme que la femelle n'éprouverait même pas le désir de l'union sexuelle si elle n'avait pas des testicules et de la semence (ibid.p. 175). Il insiste par ailleurs sur le fait que le désir sexuel chez la femme comme chez l'homme est produit par l'accumulation et l'expulsion d'une semence suite à un spasme. 
La nature a donné à tous les animaux des organes pour la conception, et elle

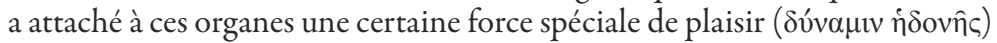
pour la génération, elle a rempli l'âme de l'être qui doit en user d'un désir étonnant et inexprimable de leur usage, en sorte que, surexcités, aiguillonnés par ce désir, les animaux, bien que dénués de raison et de toute espèce d'entendement, bien que jeunes, pourvoient à la perpétuité de la race, comme s'ils étaient complètement raisonnables ${ }^{72}$. (Trad. Daremberg.)

Le plaisir fait donc partie des «facultés naturelles » propres aux organes du corps animal, en vertu desquelles ces derniers peuvent réaliser leurs fonctions. En vertu du plaisir, en effet, les animaux sont poussés à s'accoupler. Il le font, affirme Galien, comme s'ils agissaient de façon intentionnelle, en vue de la conservation de leur espèce. De ce point de vue, il faut admettre que le plaisir est nécessairement lié aux organes reproductifs et contribue essentiellement à la reproduction.

La même explication est fournie et élargie au cas du plaisir féminin, d'abord au chap. 3, puis au chap. 9 du même livre. Dans le chap. 3, Galien reprend l'explication fournie par Aristote dans le $G A$ et précise que la conséquence immédiate du plaisir chez la femme est celle de détendre le col de l'utérus :

[...] dans le coït, <l'utérus > se dilate et se tend de telle sorte que le sperme porté à travers une voie large et facile pénètre aisément au fond des matrices ${ }^{73}$.

D’un point de vue mécanique, le plaisir sexuel féminin permet donc la dilatation du col de l'utérus, facilitant le passage de la semence masculine. Galien n'ajoute ici rien d'original par rapport à l'explication du $G A$. Il le fait toutefois par la suite lorsque il s'efforce d'intégrer cette même explication dans le cadre de ses nouvelles découvertes anatomiques. Au chap. 9 du même livre, Galien revient sur la question du plaisir, mais il assure vouloir en expliquer « la cause matérielle et organique » :

Pourquoi une très vive jouissance est-elle attachée à l'usage des parties génitales, pourquoi chez tous les animaux arrivés à la vigueur de l'âge cet usage est-il précédé d'un désir furieux ? C'est ce que nous allons dire. Nous ne recherchons pas la cause première et principale, car nous avons dit précédemment que la nature a imaginé ( $\dot{\mu} \mu \eta \chi \alpha v \mid ́ \sigma \alpha \tau o)$ ces moyens pour assurer l'éternelle jeunesse et la perpétuité de l'espèce ; il s'agit de la cause matérielle et organique. Si ce désir, si cette jouissance existent chez les animaux, ce n'est pas seulement parce que les dieux créateurs de l'homme ont voulu leur inspirer un violent désir de l'acte vénérien, ou attacher à son accomplissement une

72. Galien, De usu partium corporis humani, XIV = Kühn IV 142-210. Trad. Daremberg dans Pichot 1994.

73. Galien, De usu partium corporis humani, XIV = Kühn IV 146, 12-15. Trad. Daremberg dans Pichot 1994, p. 256. 
vive jouissance ; mais parce qu'ils ont disposé la matière et les organes pour obtenir ces résultats ${ }^{74}$.

Galien distingue ainsi deux ordres d'explication : la cause première et prin-

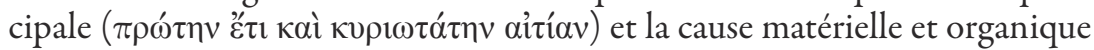

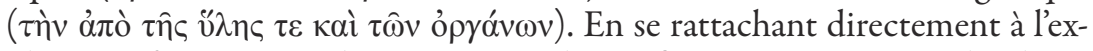
plication fournie aux chap. 2 et 3, Galien affirme à nouveau que le plaisir sexuel existe pour garantir la perpétuité de l'espèce et qu'il se produit de la même manière chez les hommes que chez les femmes. Il assure ainsi que cette explication est celle par la cause première et principale. Il pose ensuite que la cause matérielle et organique du plaisir sexuel réside dans le fait de posséder des organes reproductifs similaires ${ }^{75}$.

Galien explique ainsi que le liquide spermatique produit en excès et refluant dans l'utérus a la fonction, ou mieux, l'utilité ( $\chi \rho \varepsilon i ́ \alpha)$ de produire du plaisir. C'est en effet par sa qualité âpre et acide que ce liquide stimule les parties à agir en produisant chez la femme le plaisir qui s'achève dans son évacuation :

Cette humeur devait, outre l'utilité précédemment indiquée, en présenter une autre très importante, parce qu'elle a une âcreté et un mordant d'une nature très propre à exciter à l'usage des parties et à procurer une jouissance dans l'accomplissement de leurs fonctions ${ }^{76}$.

En reprenant les précisions données au chap. 7, Galien précise ensuite qu'en réalité la production de cette surabondance spermatique a chez la femme une double utilité, celle de produire l'excitation sexuelle et celle de refroidir les organes internes situés à gauche ${ }^{77}$.

La cause matérielle et organique permet donc d'expliquer l'existence du plaisir sexuel féminin, sans éliminer la cause première et principale. En revanche, affirme Galien, il faut admettre que « la cause organique » contribue à la réalisation et à l'existence même de « la cause primaire ».

En essayant là aussi de se rattacher aux explications du $G A$ d'Aristote, Galien conclut que le plaisir chez la femme, comme chez l'homme, est intensifié et devient « extraordinaire et excessif » lorsque dans l'émission s'ajoute au liquide spermatique du pneuma:

74. Galien, De usu partium corporis humani, XIV = Kühn IV 179, 15-180, 8. Trad. Daremberg dans Pichot 1994, p. 279.

75. Galien, De usu partium corporis humani, XIV = Kühn IV 179, 15-180, 8. Trad. Daremberg dans Pichot 1994, p. 279.

76. Galien, De usu partium corporis humani, XIV = Kühn IV 181, 3-8. Trad. Daremberg dans Pichot 1994, p. 280.

77. Galien, De usu partium corporis humani, XIV = Kühn IV 182, 9-13 : « [...] ces superfluités séreuses devant avoir une double utilité, la première d'augmenter le froid des parties gauches, la seconde d'attacher à l'usage des organes un puissant désir et une vive jouissance » (Pichot 1994, p. 280). 
S'il faut en preuve des grandes et admirables œuvres de la nature apporter de petits et misérables exemples pour éclaircir notre explication, songez qu'à l'égard de ces humeurs séreuses échauffées, il s'opère quelque chose de semblable à ce qui arrive souvent par suite de l'amas sous-cutané d'une humeur mordicante, dont le mouvement excite un titillement et une démangeaison agréable. Lors donc qu'il existe non pas seulement une humeur semblable qui a besoin d'être évacuée et qui, en conséquence, stimule et pousse à l'excrétion, mais encore un pneuma abondant et chaud, lequel demande à s'exhaler, on doit penser que la jouissance doit être excessive et incroyable ${ }^{78}$.

Comme dans le $G A$, le plaisir sexuel et son achèvement découlent donc, d'après Galien, de la production et de l'expulsion d'un liquide pneumatisé. À la différence d'Aristote, toutefois, Galien précise que ce plaisir, la présence des ovaires et la production d'un liquide spermatique ne sont pas chez la femme le produit d'une simple finalité dérivée, mais ils sont le résultat de l'œuvre absolument nécessaire de la nature, laquelle garantit qu'au plaisir sexuel, dans les deux sexes, soient associés des organes productifs et un liquide absolument analogue : les ovaires et la semence féminine.

\section{2. Complexion et finalité : Averroès sur la double causalité du plaisir}

L'idée que l'existence d'un plaisir sexuel féminin soit essentiellement liée à la production d'une semence ainsi que la thèse avancée à ce propos par Galien ont été étudiées et rejetées, dans le péripatétisme arabe, par Averroès. Dans sa paraphrase du De Generatione Animalium d'Aristote, écrite aux alentours des années soixante-dix du XII ${ }^{\mathrm{e}}$ siècle, Averroès s'interroge sur l'existence du plaisir sexuel chez les femmes et du résidu produit concomitamment ${ }^{79}$. En s'efforçant de fonder ses remarques sur des données empiriques, mais sans s'appuyer sur des observations anatomiques personnelles, Averroès va tenter de réfuter la thèse de Galien et revenir à une explication téléologique « indirecte $\gg$ du plaisir sexuel féminin.

Dans sa paraphrase à $G A$ I 20, Averroès affirme avoir questionné des femmes de confiance, qui lui ont confirmé qu'elles n'ont pas eu de plaisir, lors

78. Galien, De usu partium corporis humani, XIV = Kühn IV 181, 8-182, 2. Trad. Daremberg dans Pichot 1994, p. 280.

79. Les passages du commentaire d'Averroès, perdu en arabe, sont tirés de la traduction latine (réalisée à la Renaissance par I. Mantino) de la version arabo-hébraïque, composée par Jacob ben Makhir ibn Tibbon en 1302. Cette traduction se trouve dans l'édition des œuvres complètes d'Aristote, accompagnée du commentaire d'Averroès, réalisée par les frères Giunta : cf. Aristotelis De partibus animalium et De generatione animalium, cum Averrois Cordubensis Paraphrasi, Iacob Mantino Hispano Hebraeo, Medico, interprete, dans Aristotelis Opera cum Averrois Commentariis, vol. VI, part. 1, f. 117r-203v et vol. VI, part. 2, f. 43v-144r (dorénavant Averroès In $G A$ ), Venetiis apud Junctas, 1562-1574 (réimpr. 14 vol., Minerva, Frankfurt am Main, 1962). 
de l'union sexuelle qui a été à l'origine de leur grossesse ${ }^{80}$. Il explique alors que le plaisir sexuel, ainsi que la production concomitante d'un liquide spermatique, relèvent moins de la nature de la femme en général que de la constitution de certaines d'entre elles :

J'ai moi-même questionné des femmes, auxquelles j'accordais ma confiance, pour qu'elles me racontent la vérité, et elles m'ont confirmé cela $<i . e$. qu'elles ont engendré sans plaisir $>$. Mais certaines femmes éprouvent du plaisir dans la copulation, <tandis que d'autres ne l'éprouvent pas> en raison de leur nature sèche, surtout les femmes noires. En effet, l'humidité se trouve fréquemment dans les femmes blanches non poilues, mais dans celles dont la complexion est sèche <l'humidité $>$ est moindre ${ }^{81}$.

Comme pour ses prédécesseurs, la question n'est pas pour Averroès de nier en général le plaisir sexuel des femmes, mais de comprendre pourquoi la génération peut dans certains cas se produire sans l'émission d'un liquide et sans plaisir. Cette absence, explique alors Averroès, est due à la nature de la complexion (complexio $/{ }^{*}$ mizăğ $)$ qui est propre aux différentes espèces et aux divers individus de ces espèces.

Averroès relit ainsi l'explication qu'Aristote avait fournie en $G A$ I $20^{82}$ à la lumière de sa théorie de la complexion ${ }^{83}$ : le plaisir et l'émission d'un liquide, ou leur absence, dépendent de la complexion plus ou moins sèche des différentes femmes. Si dans la complexion la qualité de la sécheresse prévaut, l'organisme n'évacue pas d'humidité et n'éprouve pas le plaisir qui accompagne cette évacuation. La présence du plaisir et du liquide spermatique n'est donc pas le signe du fait que les femmes contribuent avec ce liquide à la formation de l'embryon, mais d'un type particulier de complexion.

Dans ce passage, donc, Averroès affirme sans hésitation qu'il y a un plaisir féminin, mais il l'analyse d'un point de vue exclusivement physiologique, en l'expliquant comme le produit d'une nécessité matérielle découlant du fait de posséder un certain type de complexion. On pourrait ainsi croire qu'Averroès n'accorde à ce phénomène qu’une nécessité purement matérielle. Le rôle causal de la complexion est d'ailleurs invoqué dans d'autres passages de la paraphrase. Cependant, lorsqu'on considère l'ensemble de ces affirmations,

80. Pour plus de précisions sur les cas considérés par Averroès, voir le texte en correspondance de la n. 90 .

81. Averroès, In GA I, f. 64, C13-D9.

82. Aristote, $G A$ I 20, 728 a1-5 : « [l'humidité produite lors de l'acte sexuel], c'est une humidité de cette région du corps propre à chaque femelle, car c'est une excrétion de l'utérus et elle se produit chez certaines, mais pas chez d'autres. Elle se produit la plupart du temps chez celles qui ont le teint pâle et sont féminines et ne se rencontre pas chez celles qui ont la peau noire et sont masculines. Sa quantité, chez les femelles chez lesquelles elle se produit quelquefois, n'est pas en rapport avec l'émission de sperme mais la dépasse de beaucoup ».

83. Sur l'importance de cette notion dans la physique averroïste, je me permets de renvoyer encore à Cerami 2015, p. 474-534. 
on comprend que si Averroès insiste sur l'aspect matériel du phénomène, c'est pour des raisons polémiques. S'il met l'accent sur la nécessité matérielle du plaisir féminin, c'est que cela lui permet de réfuter la thèse galénique de la double semence. C'est la réfutation de cette thèse qui est le véritable enjeu du phénomène à expliquer :

Tels sont les arguments par lesquels Aristote prouve que le sperme de la femme n'a pas de puissance générative, que ce soit par un mélange avec la semence de l'homme et avec le sang, ou comme Galien le pense. De fait, ce résidu selon < Aristote> procède de la nécessité du lieu, à savoir de sa nature. La femme qui le possède, en effet, lorsqu'elle émet cette humidité et éprouve du plaisir dans son expulsion est comme un jeune garçon qui est proche de l'âge de la fertilité, mais qui n'est tout de même pas encore fertile. Et cela à cause de la froideur de sa complexion et de la faiblesse de sa chaleur ${ }^{84}$.

De façon encore une fois très aristotélicienne, Averroès nous dit que le cas de la femme est comparable à celui des jeunes gens qui ont du plaisir, mais ne produisent pas de semence. Les femmes éprouvent bien du plaisir et une jouissance et elles le font au moment où elles émettent un liquide, mais ce liquide n'est pas fertile, en raison de la complexion qui est propre à la nature de la femme.

Averroès relit donc à nouveau l'affirmation d'Aristote à la lumière de sa théorie de la complexion : la complexion de la femme est trop froide pour qu'elle puisse produire une semence. Dans ce cas aussi, le plaisir féminin semble plus tenir à une nécessité matérielle qu’à une véritable finalité, mais dans ce cas aussi l'explication matérielle suffit à prouver que Galien avait tort d'affirmer que l'embryon est le produit de la semence qui procède des deux sexes et du sang menstruel.

Que Galien soit la cible principale de la paraphrase de GA I 19-23 est confirmé par la structure même du texte d'Averroès. Après avoir commenté rapidement le chapitre I 19 et le début de I 20, Averroès expose et réfute tous les arguments que Galien, dans son De Semine, avait avancés en faveur d'une explication analogique des fonctions reproductives des femmes et des hommes ${ }^{85}$. Dans ce cadre, on l'a vu, le plaisir féminin constitue l'une des preuves en faveur de la thèse qui fait du liquide spermatique féminin une semence.

Comme Aristote l'avait fait contre les défenseurs de la théorie de la double semence, Averroès utilise l'explication matérielle pour montrer, contre Galien, que l'existence d'un plaisir féminin ne prouve pas que les femmes produisent une semence analogue à celle de l'homme. À la différence d'Aristote, toutefois, Averroès se trouve face à un argument fondé sur l'observation anatomique

84. Averroès, In GA I, f. 64, E12-F10.

85. Averroès avait accès au De Semine soit par une traduction arabe attribuée à Hubayš soit par un abrégé que les sources biobibliographiques attribuent à Ḥunayn ibn Isḥāq. 
qu'il ne peut pas contourner. C'est pour répondre à cet argument qu'Averroès se trouve obligé de formuler une explication finaliste non seulement du plaisir féminin, mais aussi de l'existence des ovaires.

Concernant l'argument de Galien et l'hypothèse selon laquelle si les femmes ne produisaient pas de semence, les ovaires seraient inutiles et la nature aurait fait quelque chose en vain, Averroès avance, dans sa paraphrase, une série d'arguments visant tous à montrer que les supposés « testicules féminins » et le plaisir sexuel de la femme ont bien une fonction, mais non pas celle que leur attribuait Galien.

Bien que les deux organes sexuels, c'est-à-dire les testicules masculins et les ovaires, puissent avoir une forme similaire, selon Averroès, leur opération n'est pas la même. En utilisant un exemple dont Galien s'était également servi, Averroès explique qu'il en va de même avec les testicules de la femme et de l'homme qu'avec les seins. La ressemblance des seins des hommes avec ceux des femmes n'implique pas que les deux parties servent dans les deux cas à produire du lait. De la même manière, la production d'un liquide spermatique et les testicules qui la réalisent n'ont pas dans la femme et dans l'homme la même fonction. Chez l'homme en effet ils servent à produire une semence qui est prête à engendrer, chez la femme, en revanche, à extraire l'humidité dont l'expulsion produit le plaisir nécessaire pour pousser la femme vers la copulation et préserver ainsi l'espèce :

Galien lui-même admet que les seins de la femme sont faits pour le lait, alors que ceux de l'homme ne sont pas pour le lait, mais servent à autre chose. Jugez donc maintenant ce qui interdit qu'il en aille de même avec les testicules de la femme et de l'homme qu'avec les seins, à savoir que l'action $<$ des testicules > de l'homme et de la femme ne soit pas la même, et que leur opération dans l'homme consiste à générer une semence prête à engendrer et chez la femme à extraire l'humidité susceptible d'être extraite, pour produire le plaisir nécessaire à la copulation, de sorte qu'en cela l'espèce soit conservée ${ }^{86}$.

Comme Galien lui-même l'avait fait, Averroès admet ainsi que le plaisir de la femme a une véritable fonction dans la reproduction et la sauvegarde de l'espèce ${ }^{87}$. L'expulsion d'un liquide spermatique rend l'union avec l'homme plaisante et contribue donc indirectement à la conservation de l'espèce. Cela, rétorque toutefois Averroès, ne nous oblige pas à inférer un lien direct entre la fonction des ovaires et la production d'une semence. Les ovaires jouent bien un rôle dans la reproduction, mais non pas dans la production d'une semence.

86. Averroès, In GA I, f. 64, M-65, A9.

87. Cela ne veut pas forcément dire que les femmes de complexion sèche n'ont pas de plaisir, mais que leur plaisir est moins fréquent ou plus difficile à obtenir. En ce sens, l'explication d'Averroès n'est pas réduite aux seules femmes de complexion humide, mais à toute femme de façon générale. 
D'autres passages de la paraphrase du $G A$, ainsi que de son traité médical sur Les généralités de la médecine (Kitāb al-Kullīyyāt fì-l-tibb), connu dans le monde latin sous le titre de Colliget, confirment que le phénomène du plaisir relève pour Averroès d'une nécessité à la fois matérielle et téléologique ${ }^{88}$. Toujours dans la paraphrase, quelques lignes après avoir comparé le plaisir des femmes à celui des jeunes garçons, Averroès confirme en effet que la femme expulse elle aussi un liquide résiduel à cause du mouvement et du chatouillement qui a lieu pendant l'acte sexuel. Il explique ainsi que cette expulsion est certes de l'ordre de la nécessité matérielle, mais qu'il a également une certaine finalité : l'expulsion de ce liquide en effet rend manifeste le désir de la femme :

$<$ La femme $>$ expulse toutefois ce type de résidu à cause du mouvement et de la titillation qui se produit à cet endroit. Et < Aristote $>$ veut dire que ce résidu est donné aux femmes afin qu'il soit manifeste, outre le fait que cela se produit par la nécessité de la matière, que <les femmes> désirent l'acte sexuel, désir qui s'éteint avec l'évacuation de ce résidu ${ }^{89}$.

Dans ce passage, comme dans celui cité plus haut, la production d'un liquide spermatique n'est pas sans finalité. Elle rend manifeste le désir que la femme éprouve d'accomplir l'acte sexuel.

Une autre explication également finaliste se trouve dans les Kulliyāt, dans le chapitre consacré à l'utilité des organes sexuels. Dans la partie de ce chapitre consacrée aux vertus des organes sexuels féminins, Averroès commence par fournir contre Galien d'autres preuves du fait que la femme peut engendrer sans plaisir ${ }^{90}$. Il explique ainsi que chez la femme la vertu reproductive

88. Ce texte a vraisemblablement été écrit entre 1162 et 1169 . Averroès y est toutefois revenu par la suite, pour préciser ses explications et ajouter des passages. Cette version révisée n'est préservée que dans la traduction latine, réalisée à Padoue en 1285 par le savant juif Bonacosa et éditée à Venise en 1574. La version arabe est éditée dans Forneas Besteiro \& Álvarez de Morales (éd.) 1987. Sur la production médicale d'Averroès, voir Chandelier à paraître.

89. Averroès, In GA, f. 64, G2-10.

90. Averrois Cordubensis Colliget libri VII, dans Aristotelis Opera cum Averrois Commentariis, op. cit. supra n. 79, vol. X, suppl. I, f. 22v : « Et si tu demandes à partir de quoi il devient manifeste que le liquide spermatique de la femme ne contribue pas à la génération, il faut répliquer que cela est manifeste aux sens et connu par des arguments tirés des sens. En effet, l'homme voit que la femme tombe enceinte, même quand elle ne produit pas de liquide spermatique. Et après avoir lu les livres d'Aristote, j'ai moi-même questionné à ce propos un grand nombre de femmes qui m’ont répondu que plusieurs d'entre elles étaient tombées enceintes sans émettre de liquide spermatique et même si l'union sexuelle leur avait été déplaisante. J'ai en outre vu plusieurs femmes tomber enceintes après avoir été violées par des hommes. Et une femme qui m'est proche, à laquelle nous pouvons croire absolument, a juré sur son âme qu'elle était tombée enceinte après avoir été dans un bain chaud dans lequel des hommes malfaisants avaient éjaculé lorsqu'ils s'y étaient baignés. J'ai également analysé le livre qu’Avencladis a écrit sur la semence. J'ai constaté qu'il jugeait la chose possible; et les diverses raisons qu'il en a données m'ont plu. En effet, l'utérus attire la semence < de l'homme $>$ grâce à une puissance qui lui est propre, qu'il partage avec toute l'espèce. Or pour cela le plaisir n'est pas nécessaire. Oh, comme cela me plaît ! ». La version arabe ne rapporte pas le récit du bain. 
n'appartient pas aux testicules, mais à l'utérus.

Dans le chapitre consacré aux accidents qui peuvent survenir aux organes sexuels, Averroès explique que l'utérus a été créé pour deux raisons : en vue de la génération et comme voie d'évacuation des liquides résiduels. En raison de cela, il possède plusieurs vertus qu'Averroès énumère dans le même chapitre. La principale de ces vertus, au moins du point de vue la conception, est celle d'attirer la semence de l'homme à l'intérieur (virtus attractiva/*al-quwwa $a l-g \breve{a} \bar{d}(i b a)^{91}$. Là aussi Averroès reprend une notion galénique ${ }^{92}$, mais la réinscrit dans sa lecture aristotélicienne de la génération. L'utérus, comme d'ailleurs Aristote semblait aussi l'envisager, possède donc en lui la capacité d'attirer la semence de l'homme. Or, conclut Averroès, pour que cette fonction s'active, il n'y a nul besoin que la femme éprouve du plaisir. C'est donc cette vertu qui est propre à l'utérus dans son ensemble et non pas aux ovaires, qui sont absolument nécessaires à la conception.

Concernant le liquide spermatique et les supposés « testicules féminins », Averroès explique alors que leur fonction n'est pas stricto sensu reproductive, mais qu'elle possède tout de même une utilité. Averroès affirme que le liquide résiduel féminin a une fonction analogue à celle de la salive, par rapport à l'ingestion de la nourriture :

[...] Toutes ces choses montrent que le liquide spermatique de la femme est un résidu qui s'écoule en vue de la délectation, comme s'écoule la salive de la bouche de quelqu'un qui a faim, lorsqu'il voit quelqu'un d'autre manger ${ }^{93}$.

Cette analogie confirme donc que le liquide spermatique et le plaisir féminins possèdent pour Averroès une certaine utilité associée indirectement à une fonction vitale, celle de la reproduction. Le liquide qui accompagne le plaisir féminin n'est pas stricto sensu nécessaire à la production de l'embryon, mais rend plaisante la copulation et facilite l'action que la femme doit accomplir pour contribuer à la génération.

Comme on l'a fait pour Aristote, on peut donc conclure que le phénomène du plaisir féminin, ainsi que la production du liquide qui l'accompagne, ne sont pas en tant que tels en vue d'une fin, mais en vue du meilleur. L'explication proposée en $G A$ II 4, toutefois, ne pouvait plus satisfaire Averroès, qui se trouvait devoir expliquer l'existence d'un organe dont Aristote ignorait l'existence et auquel depuis Galien on attribuait la production du liquide à l'origine du plaisir. Sa stratégie consiste alors à attribuer l'explication en vue

91. L'utérus a notamment quatre vertus : attractiva, retentiva, digestiva (voire conservativa) et expulsiva.

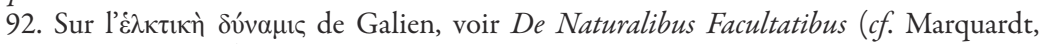
Mueller \& Helmreich (éd.) 1884-1893, III, p. 216, 17-24 = Kühn II, p. 159-161). Sur la présence de cette notion chez Alexandre d'Aphrodise, voir Quaestio II 23, 74.14-17. Sur la lecture que ce dernier a pu en faire, voir Rashed (éd.) 2015, p. 171 sq.

93. Averrois Cordubensis Colliget, op. cit. supra note 90, f. 23r. 
du meilleur à l'organe qui d'après cette thèse est à l'origine du liquide et du plaisir qui en découle : les testicules féminins.

L'hypothèse que cet organe est selon Averroès non pas nécessaire, mais en vue du meilleur est confirmée par le fait qu'Averroès affirme très clairement que, dans le cas des mâles qui les possèdent, l'existence des testicules s'explique par ce type de raison. Suivant en cela le propos d'Aristote, Averroès affirme en effet que les testicules existent, non pas parce qu'ils sont nécessaires, mais parce que, dans le cas de certaines espèces, il est mieux de les posséder ${ }^{94}$.

L'embryon donc n'a pas à être produit par deux matières ou par deux agents : il n'a qu'une seule forme, une seule matière et un seul agent. Dans le respect de ce principe aristotélicien, Averroès conclut alors que c'est la femme qui donne la matière, le sang menstruel, et que c'est l'homme qui lui donne la forme en agissant par sa semence. La nature et l'ontologie aristotélicienne sont ainsi sauvées. Contre Galien, Averroès peut affirmer que la nature ne fait rien en vain, parce que même si le plaisir sexuel féminin n'est pas directement en vue de la procréation, il existe tout de même parce qu'il est mieux que ce soit ainsi.

\section{Conclusion}

Le plaisir sexuel féminin, comme tout plaisir corporel, ne se réduit pas simplement aux mécanismes biologiques qui le produisent et l'accompagnent. Nombreux sont les passages du corpus aristotélicien qui présentent le plaisir sexuel comme une motivation très puissante qui pousse l'animal à l'action ${ }^{95}$. Dans ces contextes, Aristote n'hésite pas à affirmer que l'animal en général et la femme en particulier sont poussés à s'accoupler en vue du plaisir sexuel, considéré pour cela même comme une fin en soi qui conduit à la conservation de l'espèce. Ce n'est pas dans cette perspective que j'ai décidé de considérer ici le plaisir sexuel féminin, mais en l'examinant dans sa complexité biologique, dans le but de comprendre la place qu'Aristote lui attribue dans son cadastre étiologique. Je suis toutefois convaincue que les résultats de l'analyse que j’en ai proposée non seulement ne sont pas incompatibles avec cet aspect de la question, mais qu'ils fournissent des éléments essentiels pour le comprendre.

Dans la première partie de cet article, j’ai suggéré que le plaisir sexuel féminin et la production du liquide spermatique qui l'accompagne font partie des phénomènes qu'Aristote définit comme « en vue du bien » et « pour le meilleur ». Le plaisir sexuel, en effet, n’est ni « nécessaire », ni « d'une accidentalité utile », mais se produit chez les femmes « le plus souvent » et il a pour fonction de faciliter l'activité de la partie assignée de

94. Sur le débat qu'Averroès engage sur cette question contre Galien et sur les passages qui en témoignent, voir Cerami 2015 chap. VIII, p. 480-487.

95. Cf. notamment $H A$ VI 18, 571 b9-10 ; VII 1, 581 b11-21. Mais voir aussi, à ce propos, le Problème 4, 878 b. 
façon première à la procréation, à savoir l'utérus. Lors de la jouissance, en effet, le col de l'utérus se desserre, facilitant ainsi l'une des fonctions propres de ce dernier, à savoir l'attraction de la semence du mâle. Par conséquent, en tant que phénomène en vue du meilleur, le plaisir sexuel féminin doit être considéré comme la condition naturelle à laquelle l'individu est disposé et dans laquelle par nature il doit se trouver.

L'étude de ce phénomène a ainsi donné l'occasion de s'interroger à nouveaux frais sur la question plus générale de l'articulation entre les différentes formes de finalité d'une part, et entre la causalité formalo-finale et la causalité matérielle d'autre part. Sans nier l'existence d'une « nécessité de la matière $\gg$ et son autonomie relative eu égard aux propriétés découlant directement de la forme, j'ai suggéré que le plaisir sexuel féminin et les autres phénomènes en vue du mieux ne doivent pas être considérés comme des effets « indépendants » de la forme de l'animal, mais comme des conséquences indirectes de celle-ci. Plus précisément, j’ai formulé l'hypothèse que la production de résidu est, dès l'information de la matière, potentiellement inscrite dans le projet général que la forme dicte.

Cette hypothèse interprétative permet d'éclairer le statut même de ces phénomènes et fournit l'enjeu ultime de la distinction aristotélicienne entre l'explication en vue du nécessaire et l'explication en vue du meilleur. En effet, les phénomènes en vue du mieux sont non nécessaires, non pas parce qu'ils ne sont pas inscrits dans la forme de l'animal, mais dans la mesure où ils permettent de fixer le dernier niveau d'accomplissement formel, auquel l'animal peut parvenir ou pas. Au niveau de la classe propre dans laquelle le phénomène en vue du mieux se manifeste, la condition d'optimalité permet de définir l'excellence vers laquelle cette classe même est orientée, dans la mesure où sa présence est le signe que l'animal en question n'a pas simplement réalisé son « être », mais son « bien être ». Au niveau générique, la condition d'optimalité se comprend également au moment où l'on compare l'espèce en question aux autres, de niveau «supérieur », dans lesquelles ce phénomène ne se manifeste pas. Le phénomène en vue du bien, en d'autres termes, se comprend comme « meilleur », pour l'espèce dans laquelle il se produit, lorsqu'on prend en compte les autres espèces de la même classe dans lesquelles le phénomène ne se produit pas. En un sens fort, donc, et à tout niveau, les phénomènes en vue du mieux ne sont pas le produit d'une téléologie «manquée », ni d'une prééminence relative de la nécessité matérielle, mais le signe du statut supérieur de l'individu, de l'espèce ou de la classe générique chez qui ils se réalisent.

Dans ce cadre, la comparaison avec Galien et le débat engagé par Averroès contre ce dernier, auxquels est consacrée la seconde partie de l'article, ont non seulement montré l'importance que la question du plaisir sexuel féminin a reçue dans la transmission du péripatétisme gréco-arabe, mais ils ont 
permis d'éclairer en retour la doctrine du Stagirite. En effet, en dépit d'une apparente affinité des affirmations de Galien dans l'Utilité des Parties et de celles d'Aristote, concernant le fait que le plaisir sexuel est une fin qui pousse à l'action, leurs théories sur le plaisir sexuel demeurent tout à fait incompatibles $^{96}$. Je vais en conclusion expliquer pourquoi.

Le différend entre Galien et Aristote ne repose ni sur une simple question d'observation anatomique, ni sur une pure question de principe. Il vient d'un usage foncièrement différent de l'analogie. En affirmant que le plaisir sexuel de la femme est absolument de même nature que celui de l'homme, car dans l'un comme dans l'autre cas il y a production de semence, Galien a établi une analogie stricte entre l'un et l'autre. D'après cette analogie, si la femme differe de l'homme, c'est notamment d'un point de vue quantitatif : la femme étant un homme amoindri, sa semence ne differe de celle de ce dernier qu'en degré de raffinement, tout comme la nature de sa complexion. Aristote, en revanche, n'a pas comparé directement les deux sexes et les propriétés qui les caractérisent ; il a établi une analogie de fonction, d'après laquelle l'homme et la femme, leur appareil reproductif et les phénomènes qui y sont liés ne sont pas qualitativement les mêmes, mais complémentaires. Ce qui ne veut pas dire que les phénomènes de la femme et de l'homme ne sont pas comparables, y compris d'un point de vue quantitatif, mais qu'ils ne le sont pas selon le plus et le moins comme les éléments d'une même classe au sens strict.

C'est précisément la finalité en vue du mieux, qu'Aristote attribue à la femme, à son existence, ainsi qu’à son plaisir sexuel, qui le confirme. En effet, comme j’ai essayé de le montrer, la condition d'optimalité qui caractérise ces phénomènes peut se comprendre dans les deux sens, c'est-à-dire comme désignant aussi bien la condition de l'individu mâle complet, et donc celle de l'espèce, que celle de la femme et de ses états physiologiques, qui sont réintégrés dans le projet général dicté par la forme et réalisent, dans ce réinvestissement, non seulement leur propre bien, mais le bien être de l'espèce. En effet, le plaisir sexuel de la femme - comme c'est le cas de la femme elle-même au niveau spécifique - est ce qui lui permet, en vertu de sa nature propre, de réaliser l'excellence de l'animal et donc son «bien être ». Loin de réduire le plaisir sexuel féminin à la production d'un lubrifiant, la doctrine aristotélicienne telle qu'on l'a présentée permet donc de dégager la valeur propre de ce phénomène et son statut pour ainsi dire métaphysique : ce plaisir, sans constituer l'essence de l'animal en général et de l'être humain en particulier, y est intrinsèquement lié.

C'est dans ce même cadre que s'inscrit la lecture qu'Averroès propose du plaisir sexuel féminin. En saisissant la particularité du paradigme aristotélicien, il met bien en lumière l'incompatibilité de ce dernier avec la doctrine

96. Je rejoins sur ce point les conclusions de Connell 2000. Je ne veux pas rentrer, en revanche, dans le débat qui porte sur le caractère plus ou moins féministe de leurs doctrines. 
galénique et s'efforce de le défendre tout en le renouvelant. En effet, en attribuant l'explication en vue du meilleur à l'organe qui d'après la thèse galénique était à l'origine de la semence féminine, à savoir les « testicules féminins », il conclut que cet organe est non pas nécessaire, mais « en vue du meilleur $\gg$ dans la mesure où il existe non pas parce qu'il produit de la semence, mais parce qu'il produit le liquide qui rend l'acte sexuel et donc la procréation plus faciles.

L'étude du débat qu'Averroès engage contre Galien sur cette question nous donne ainsi un aperçu de la richesse de la réception arabe de l'aristotélisme et confirme par cela même, à l'encontre d'une idée encore largement répandue, que la biologie aristotélicienne ne disparaît pas avec Théophraste pour réapparaître avec Albert le Grand, mais qu'elle trouve dans le Moyen-Âge arabe le terrain propice qui permettra sa transmission et son renouvellement pour les siècles qui suivent ${ }^{97}$.

97. Sur l'importance de la réception arabe dans l'histoire de la transmission du corpus biologique d'Aristote, voir Cerami \& Falcon 2014. 


\section{BIBLIOGRAPHIE}

Accattino, P. 1994 : « Galeno e la riproduzione animale : analisi nel De semine », Aufstieg und Niedergang der Römischen Welt, II, 37.2, Berlin-New York, 1994, p. $1856-1886$.

BALme, D. M. 1987 : «Teleology and Necessity », dans A. Gotthelf \& J. G. Lennox (éd.), 1987, p. 275-286.

Bianchi, E. 2014 : The Feminine Symptom : Aleatory Matter in the Aristotelian Cosmos, New York, 2014 (Società antiche).

Boylan, M. 1984 : «The Galenic and Hippocratic Challenges to Aristotle's Conception Theory », Journal of the History of Biology, 17/1 (1984), p. 83-112.

- 1986: « Galen's Conception Theory », Journal of the History of Biology, 19/1 (1986), p. 47-77.

Campbell, M. 2011 : Le plaisir dans la pensée d'Aristote : physiologie, essence, valeur et usage, Thèse de doctorat en histoire de la philosophie, Université Paris IV-Sorbonne, 2011.

Campese, S., P. Manuli \& G. Sissa 1983 : Madre Materia : Sociologia e biologia della donna greca, Torino, 1983.

Cerami, C. 2015 : Génération et Substance : Aristote et Averroès entre physique et métaphysique, Boston/Berlin, 2015 (Scientia Graeco-Arabica, 18).

- (à paraître) : « Function and Instrument: Toward a New Criterion of the Scale of Being in Aristotle's Generation of Animals $\gg$, dans A. Falcon \& D. Lefebvre (éd.), The Generation of Animals and Aristotle's philosophy, Cambridge, à paraître.

Cerami, C. \& A. Falcon 2014 : « Continuity and Discontinuity in the Greek and Arabic Reception of Aristotle's Study of Animals », Antiquorum Philosophia, 8 (2014), p. 35-56.

Chandelier, J. (à paraître) : «Averroes on Medicine », dans P. Adamson et M. Di Giovanni (éd.), Interpreting Averroes, à paraître.

Code, A. 1997 : « The Priority of Final Causes over Efficient Causes in Aristotle's Parts of Animals », dans W. Kullmann \& S. Föllinger (éd.), Aristotelische Biologie: Intentionen, Methoden, Ergebnisse: Akten des Symposions über Aristoteles Biologie vom 24.-28. Juli 1995 in der Werner-Reimers-Stiftung in Bad Homburg, Stuttgart, 1997, p. 127-143 (Philosophie der Antike, 6).

Connell, S. M. 2000 : « Aristotle and Galen on Sex Difference and Reproduction : A New Approach to an Ancient Rivalry », Studies in History and Philosophy of Science, Part A, 31/3 (2000), p. 405-427.

Cooper, J. M. 1987 : « Hypothetical Necessity and Natural Teleology », dans A. Gotthelf \& J. Lennox (éd.), 1987, p. 243-274.

De Lacy Ph. 1992 (éd.) : Galen, On semen, Berlin, 1992 (Corpus medicorum Graecorum, V, 3, 1).

Dean-Jones, L. 1992 : « The Politics of Pleasure : Female Sexual Appetite in the Hippocratic Corpus », dans D .C. Stanton (éd.), Discourses of Sexuality : From Aristotle to AIDS, Ann Arbor 1992, p. 48-77.

Deslauriers, M. 1998 : « Sex and Essence in Aristotle's Metaphysics and Biology », dans C. A. Freeland (éd.), 1998, p. 138-167.

Fórneas Besteiro, J. M. \& C. Álvarez de Morales 1987 (éd.) : Averroès, Kitāb al-Kullìyyàt fì-l-țibb (= Colliget), Madrid, 1987. 
Freeland, C. A. 1998 (éd.) : Feminist Interpretations of Aristotle, University Park (PA), 1998 (Re-Reading the canon).

Gotthelf, A. 1987 : «Aristotle's Conception of Final Causality », dans A. Gotthelf \& J. G. Lennox (éd.), 1987, p. 226-254.

- 1989: « The Place of the Good in Aristotle's Teleology », dans J. J. Cleary \& D. C. Shartin (éd.), Proceedings of the Boston Area Colloquium in Ancient Philosophy, 4, 1988, Lanham, 1989, p. 113-139.

Gotthelf, A. \& J .G. Lennox 1987 (éd.) : Philosophical issues in Aristotle's biology, Cambridge/New York, 1987.

Gutas, D. 2010 (éd.) : Theophrastus On First Principles (known as his Metaphysics): Greek Text and Medieval Arabic Translation, edited and translated with introduction, commentaries and glossaries, as well as the medieval Latin translation, and with an excursus on Graeco-Arabic editorial technique, Leiden/Boston, 2010 (Philosophia antiqua, 119).

Henry, D. 2007 : « How Sexist is Aristotle's Developmental Biology ? », Phronesis, 52/3 (2007), p. 251-269.

Jacluart, D. \& C. Thomasset 1985 : Sexualité et savoir médical au Moyen-Âge, Paris, 1985 (Les Chemins de l'Histoire, 8).

Joly, R. 1970 (éd.) : Hippocrate, Tome XI : De la génération, De la nature de l'enfant, Des maladies VI, Du foetus de huit mois, Paris, 1970 (Collection des Universités de France. Série grecque, 198).

Kosman, A. 2010 : «Male and Female in Aristotle's Generation of Animals » dans J. G. Lennox \& R. Bolton (éd.), Being, Nature, and Life in Aristotle : essays in honor of Allan Gotthelf, Cambridge, 2010, p. 147-167.

KüHN, K. G. 1821-1833 (éd.) : Galenus, De usu partium corporis humani XIV, dans Galeni opera omnia, vol. IV, Leipzig, 1822.

Laks, A. \& G. W. Most 1993 (éd.) : Théophraste, Métaphysique, Paris, 1993 (Collection des Universités de France. Série grecque, 358).

Lefebvre, D. 2014a : « La jument de Pharsale : retour sur De generatione animalium IV, 3 » dans C. Cerami (éd.), Nature \& Sagesse : les rapports entre physique et métaphysique dans la tradition aristotélicienne : recueil de textes en hommage à Pierre Pellegrin, Leuven, 2014 (Aristote, traductions et études), p. 207-271.

- $2014 \mathrm{~b}$ (trad.) : Aristote, La génération des animaux, dans P. Pellegrin (éd.), Aristote: CEuvres complètes, Paris, 2014, p. 1575-1730.

Lennox, J. G 1987 : « Divide and Explain : The Posterior Analytics in Practice », dans A. Gotthelf \& J. G. Lennox (éd.), 1987, p. 190-119.

— 1997a : « Material and Formal Natures in Aristotle's De Partibus Animalium », dans J. J. Cleary \& W. Vians (éd.), Proceedings of the Boston Area Colloquium in Ancient Philosophy, 11, 1995, Lanham, 1997, p. 217-240.

- 1997b : « Nature Does nothing in Vain... », dans H.-.C. Günther \& A. Rengakos (éd.), Beiträge zur antiken Philophie : Festschrift für Wolfgang Kullmann, Stuttgart, 1997, p. 199-214.

Leunissen, M. 2010 : Explanation and Teleology in Aristotle's Science of Nature, Cambridge, 2010.

Lloyd, E. 2005 : The Case of the Female Orgasm: Bias in the Science of Evolution, Cambridge (Mass.), 2005. 
Louguet, C. 2015 : « Aristote et les théories pangénétiques du v siècle : enjeux métaphysiques d'un débat biologique », dans V. Gysembergh \& A. Schwab (éd.), Le Travail du Savoir = Wissensbewältigung: Philosophie, sciences exactes et sciences appliquées dans l'Antiquité, Trier, 2015 (AKAN-Einzelschriften, 10), p. 119-161.

Mayhew, R. 2004 : The Female in Aristotle's Biology : Reason or Rationalization, Chicago/Londres, 2004.

Marquardt, I., I. Mueller \& G. Helmreich 1884-1893 (éd.) : Cl. Galeni Pergameni Scripta minora, 3 vol., Leipzig, 1884-1893.

MORSINK, J. 1982 : Aristotle on the generation of animals : A philosophical study, Washington, 1982.

Nielsen, K. 2008 : « The Private Parts of Animals : Aristotle on the Teleology of Sexual Difference », Phronesis, 53/4 (2008), p. 373-405.

Pellegrin, P. 1986 : « Les fonctions explicatives de l'Histoire des animaux d'Aristote », Phronesis, 31/1 (1986), p. 148-166.

- 1990 : «De l'explication causale dans la biologie d'Aristote », Revue de métaphysique et de morale, 95/2 (1990), p. 197-219.

- 2002 : «Les ruses de la nature et l'éternité du mouvement : encore quelques remarques sur la finalité chez Aristote », dans M. Canto-Sperber \& P. Pellegrin (éd.), Le style de la pensée : recueil de textes en hommage à Jacques Brunschwig, Paris, 2002, p. 296-323.

- 2011 (trad.) : Aristote, Les parties des animaux, traduction, présentation et notes, Paris, 2011 (G. F., 1187).

Pichot, A. 1994 (éd.) : Galien, CEuvres médicales choisies, Tome 1, traduction de Ch. Daremberg, choix, présentation et notes par A. Pichot, Paris, 1994 (Tel, 235).

Preus, A. 1977 : « Galen's Criticism of Aristotle's Conception Theory », Journal of the History of Biology, 10/1 (1977), p. 65-85.

Rashed, M. 2002 : « La préservation ( $\sigma \omega \tau \eta \rho i ́ \alpha)$, objet des Parva naturalia et ruse de la nature $\gg$, Revue de philosophie ancienne, 20/1 (2002), p. 35-59.

- 2015 (éd.) : Al-Hasan ibn Müsā al-Nawbakhtī, Commentary on Aristotle's De generatione et corruptione, Berlin/New York, 2015 (Scientia GraecoArabica, 19).

WitT, C. 1998 : « Form, Normativity, and Gender in Aristotle : A Feminist Perspective », dans C. A. Freeland (éd.), 1998, p. 118-137. 